\title{
Theory of Type-II Superconductors with Finite London Penetration Depth
}

\author{
Ernst Helmut Brandt \\ Max-Planck-Institut für Metallforschung, D-70506 Stuttgart, Germany
}

(October 29, 2018)

\begin{abstract}
Previous continuum theory of type-II superconductors of various shapes with and without vortex pinning in an applied magnetic field and with transport current, is generalized to account for a finite London penetration depth $\lambda$. This extension is particularly important at low inductions $B$, where the transition to the Meissner state is now described correctly, and for films with thickness comparable to or smaller than $\lambda$. The finite width of the surface layer with screening currents and the correct dc and ac responses in various geometries follow naturally from an equation of motion for the current density in which the integral kernel now accounts for finite $\lambda$. New geometries considered here are thick and thin strips with applied current, and "washers", i.e. thin film squares with a slot and central hole as used for SQUIDs.
\end{abstract}

PACS numbers: 74.60.Ec, 74.60.Ge, 74.55.+h

\section{INTRODUCTION}

The statics and dynamics of the Abrikosov vortex lines and of the two dimensional (2D) pancake vortices in layered superconductors lated within continuum approximation in terms of an equation of motion of the current density $\mathbf{j}$ inside the superconductor. 3 The resulting integral equation for the space and time dependent $\mathbf{j}(x, y, z, t)$ contains the assumed constitutive law for the electric field $\mathbf{E}_{v}$ caused by the motion of vortices. In general, the law $\mathbf{E}=\mathbf{E}_{v}(\mathbf{j}, \mathbf{B})$ depends on the current density $\mathbf{j}$ and on the local induction $\mathbf{B}$ and describes, e.g., free flux flow, pinning, and thermally activated depinning of vortices.

Besides this, the equation of motion for $\mathbf{j}$ depends on the geometry of the given problem. Various geometries have been considered: Thin long strips 1 thin circular disks and rings 5 thin rectangular platelets or films, thick strips, always in a magnetic field applied perpendicular to the plane of the superconductor. Further numerical solutions for the statics of thin film superconductors of various shapes within the Bean model are given by Prigozhin. 8 In all these geometries, the current density $\mathbf{j}$ or the sheet current in films $\mathbf{J}(x, y, t)=\left(J_{x}, J_{y}\right)=\int \mathbf{j}(x, y, z, t) d z$ have only one component (flowing along the strip or circulating in the disk) or may be derived from a scalar potential $g(x, y, t)$ according to $J_{x}=\partial g / \partial y, J_{y}=-\partial g / \partial x$ since $\nabla \cdot \mathbf{J}=0$. The equation of motion describes thus a scalar $(j$ or $g$ ) which depends on only one or two spatial coordinates.

A more general continuum formulation including also transport currents applied by contacts, applying in principle to arbitrary three dimensional (3D) geometry, and accounting also for a finite lower critical field $H_{c 1}$ and for a general reversible thermodynamic field $H(B)$, is presented in Ref. 10, where it is applied to the problem of the geemetric barrier.11 The elegance of the formulation 6,10 in terms of an equation of motion for the current density is that it explicitly accounts for the applied magnetic field or transport current, which in other formulations enter via a separate boundary condition for a differential equation. Our integral equation automatically accounts for the inhomogeneous magnetic field outside the superconductor, without any need to calculate this infinitely extended field explicitly during the time integration since all spatial integrations extend only over the finite specimen cross section. The applied field and applied current enter via the screening currents that flow on the specimen surface, the information about the geometry is contained in a pre-calculated integral kernel, and the properties of the superconductor enter via one single constitutive law $E=E_{v}(j, B)$ if $B=\mu_{0} H$ may be assumed, or via two laws if some nontrivial law $B(H) \neq \mu_{0} H$ is used.

So far, this continuum description 1 , 10 considered the limit where both the vortex spacing $a$ and the London penetration depth $\lambda$ are assumed to be much smaller than all other relevant lengths. There are, however, two situations where a finite London depth $\lambda$ should be considered:

(i) In bulk superconductors, at small inductions $B$ the Meissner state should follow in the limit $B \rightarrow 0$. So far, with the above assumption $E=E_{v}(j, B)$ one has $E=0$ for $B \rightarrow 0$ if the free flux flow law or similar laws $E_{v} \propto B$ are used. In real type-II superconductors, however, one may have $E \neq 0$ in a surface layer of thickness $\lambda$ if $j$ varies with time, since one has $E=\mu_{0} \lambda^{2} \partial j / \partial t$ in the Meissner-London limit (this London equation means that the electric field accelerates the Cooper pairs).

(ii) In thin films with thickness $d$, both cases $d>\lambda$ and $d<\lambda$ should be described correctly by a continuum theory. In particular for $d \ll \lambda$ the effective penetration depth of thin film $122 \Lambda=\lambda^{2} / d$ may become macroscopically large and comparable with the film width.

In the present paper our previous fontinuum electrodynamics of type-II superconductors 1 \& 10 is generalized to allow for an arbitrary London penetration depth $\lambda$. The resulting integral equations for $j$ in various geome- 
tries correctly describe all limiting cases and are easily solved numerically. With finite $\lambda$ the stability and speed of the numerics is even improved since this $\lambda$ provides a well defined inner cutoff length, whereas with $\lambda=0$ the inner cutoff depends on the chosen grid spacing.

In the following sections, equations of motion for the current density in type-II superconductors with finite London depth $\lambda$ are derived for various geometries. For illustration, some selected numerical results are presented for each geometry, namely, current and magnetic field profiles, magnetization curves, ac susceptibilities, and the current stream lines in thin films.

\section{VOLTAGE-CURRENT LAW}

In type-II superconductors within continuum approximation the local electric field $E$ is composed of two parts, originating from the vortex motion and from the Meissner surface currents,

$$
\mathbf{E}=\mathbf{E}_{v}+\mathbf{E}_{M}
$$

The first term $\mathbf{E}_{v}(\mathbf{j}, \mathbf{B})$ in general is a nonlinear function of the current density $\mathbf{j}$ due to pinning and thermal or quantum depinning (creep). It may be anisotropic (a) if the Hall effect of vortex motion is accounted for, or (b) if the superconductor is anisotropic, or (c) if $\mathbf{j}$ is not perpendicular to $\mathbf{B}$. This latter case is still little understood but should occur in most really three dimensional geometries, e.g., in a superconductor cube with applied magnetic field; one possible model description in this case uses two critical current densities $j_{c \perp}$ and $j_{c \|}$ for the current components perpendicular and parallel to B. Though the parallel current density $j_{\|}$ does not exert a Lorentz force on the wprices it nevertheless can trigger a helical instability 13,14 and vortex cutting,15 which leads to a finite $j_{c \|}$. Furthermore, as shown by Gurevich 16 some combinations of nonlinearity and anisotropy in voltage-current models may lead to unstable current distributions. In the following geometry examples, for transparency I shall consider isotropic $\mathbf{E}_{v}$ since the extension to anisotropic $\mathbf{E}_{v}$ is straightforward. Isotropic voltage-current laws have the form

$$
\mathbf{E}_{v}(\mathbf{j}, B)=\rho_{v}(j, B) \mathbf{j},
$$

where $\rho_{v}(j, B)$ is the resistivity caused by vortex motion, and $j=|\mathbf{j}|, B=|\mathbf{B}|$, with $\mathbf{j} \perp \mathbf{B}$. In the free flux flow limit (zero pinning or high current density) one has $\rho_{v}=\rho_{\mathrm{ff}} \approx\left(B / B_{c 2}\right) \rho_{n}$ where $B_{c 2}$ is the upper critical field and $\rho_{n}$ the normal resistivity just above $B_{c 2}$. For thermally activated depinning many experiments and theoretical models yield a logarithmic activation energy $U(j)=U_{0} \ln \left(j_{c} / j\right)$, which gives $E_{v}=E_{c} \exp (-U / k T)=$ $E_{c}\left(j / j_{c}\right)^{n}$ with creep exponent $n=U_{0} / k T \gg 1$ and (arbitrary) "threshold criterion" $E_{c}=E\left(j_{c}\right)$. With Eq. (2) this means $\rho_{v}=\rho_{c}\left(j / j_{c}\right)^{\sigma}$ where $\sigma=n-1 \gg 1$. A simple model which combines this creep model and the free flux flow limit reads 10

$$
\rho_{v}(j, B)=\frac{\rho_{n}}{B_{c 2}} B \frac{\left(j / j_{c}\right)^{\sigma}}{1+\left(j / j_{c}\right)^{\sigma}} .
$$

The second term in Eq. (1) is caused by the current flowing in the Meissner state and is given by the dynamic London equation,

$$
\mathbf{E}_{M}=\mu_{0} \lambda^{2} \frac{\partial \mathbf{j}}{\partial t}
$$

This term describes the acceleration of the massive charge carriers (Cooper pairs) by an electric field, $\partial j / \partial t \propto E$. Thus, a finite London depth $\lambda$ in pripciple may be introduced into our continuum description 6 , 10 by using the full voltage-current law,

$$
\mathbf{E}=\mathbf{E}_{v}(\mathbf{j}, \mathbf{B})+\mu_{0} \lambda^{2} \frac{\partial \mathbf{j}}{\partial t}
$$

This replacement works well in the case of linear response, where it yields a frequency $(\omega)$ dependent complex resistivity

$$
\rho(\omega)=\rho_{\mathrm{ff}}+i \omega \mu_{0} \lambda^{2}
$$

in the absence of vortex pinning. In this case the linear integral equation for $j$ reduces to an eigen value equation from which the complex ac susceptibility of the supercop ductor for a given geometry follows as an explicit sum.17

When $E_{v}(j, B)$ is nonlinear, the integral equation for $j(\mathbf{r}, t)$ has to be solved by numerical integration over time. It turns out that this time integration becomes unstable when the modified voltage-current law (5) is inserted. However, when the term containing $\lambda^{2}$ is incorporated into the integral kernel as shown in Sct. 3, then the numerics remains stable and even becomes more stable and faster than it was with $\lambda=0$.

\section{THICK STRIPS WITH FINITE $\lambda$}

To fix ideas we first derive the modified integral equation for the geometry of an infinite strip or bar with rectangular cross section filling the volume $-a \leq x \leq a$, $-b \leq y \leq b,-L / 2 \leq z \leq L / 2, L \gg a, b$, see Fig. 1 . In the general $3 \mathrm{D}$ geometry, with $\mathbf{r}=(x, y, z)$, the vector potential $\mathbf{A}_{\mathbf{j}}(\mathbf{r})$ caused by the current density $\mathbf{j}(\mathbf{r})=-\mu_{0}^{-1} \nabla^{2} \mathbf{A}_{\mathbf{j}}$ in the gauge $\nabla \cdot \mathbf{A}=0$ is given by

$$
\mathbf{A}_{\mathbf{j}}(\mathbf{r})=\mu_{0} \int d^{3} r^{\prime} \frac{\mathbf{j}\left(\mathbf{r}^{\prime}\right)}{4 \pi\left|\mathbf{r}-\mathbf{r}^{\prime}\right|}
$$

with the integral taken over the volume where the current flows. Indeed, using $\nabla^{2}\left|\mathbf{r}-\mathbf{r}^{\prime}\right|^{-1}=-4 \pi \delta_{3}\left(\mathbf{r}-\mathbf{r}^{\prime}\right)$ ( $\delta_{3}$ is the $3 \mathrm{D}$ delta function) one verifies that Eq. (7) yields $\nabla^{2} \mathbf{A}_{\mathbf{j}}(\mathbf{r})=-\mu_{0} \mathbf{j}(\mathbf{r})$. For a long strip or bar, both 
$\mathbf{j}=j(x, y) \hat{\mathbf{z}}$ and $\mathbf{A}=A(x, y) \hat{\mathbf{z}}$ are directed along $z$. Integrating Eq. (7) over $z$ and writing from now on $\mathbf{r}=(x, y)$ we obtain

$$
A_{j}(\mathbf{r})=\mu_{0} \int d^{2} r^{\prime} j\left(\mathbf{r}^{\prime}\right) Q_{\mathrm{bar}}\left(\mathbf{r}, \mathbf{r}^{\prime}\right)
$$

with the 2D integral kernel

$$
Q_{\mathrm{bar}}\left(\mathbf{r}, \mathbf{r}^{\prime}\right)=\frac{1}{2 \pi} \operatorname{asinh} \frac{L / 2}{\left|\mathbf{r}-\mathbf{r}^{\prime}\right|} \approx \frac{1}{2 \pi} \ln \frac{L}{\left|\mathbf{r}-\mathbf{r}^{\prime}\right|} .
$$

The integral (8) is over the rectangular strip cross section, $-a \leq x \leq a$ and $-b \leq y \leq b$, but actually Eq. (8) applies to strips with cross sections of any shape. If the current distribution is symmetric the integration (8) may be restricted to one quarter of the rectangular cross section, e.g.,

$$
A_{j}(x, y)=\mu_{0} \int_{0}^{a} d x^{\prime} \int_{0}^{b} d y^{\prime} j\left(x^{\prime}, y^{\prime}\right) Q_{\mathrm{bar}}^{\mathrm{sym}}\left(x, y ; x^{\prime}, y^{\prime}\right) .
$$

For strips with a magnetic field $H_{a}$ applied along $y$, one has $j(x, y)=-j(-x, y)=j(x,-y)=-j(-x,-y)$, thus

$$
Q_{\mathrm{bar}}^{\mathrm{sym}}=Q_{\mathrm{bar}}^{H}=\frac{1}{4 \pi} \ln \frac{\left(x_{+}^{2}+y_{-}^{2}\right)\left(x_{+}^{2}+y_{+}^{2}\right)}{\left(x_{-}^{2}+y_{-}^{2}\right)\left(x_{-}^{2}+y_{+}^{2}\right)}
$$

with $x_{ \pm}=x \pm x^{\prime}, y_{ \pm}=y \pm y^{\prime}$. For strips with transport current $I_{a}$ along $z$ one has $j(x, y)=j(-x, y)=$ $j(x,-y)=j(-x,-y)$, thus

$$
\begin{aligned}
& Q_{\mathrm{bar}}^{\mathrm{sym}}=Q_{\mathrm{bar}}^{I}= \\
& \frac{1}{4 \pi} \ln \frac{L^{8}}{\left(x_{-}^{2}+y_{-}^{2}\right)\left(x_{-}^{2}+y_{+}^{2}\right)\left(x_{+}^{2}+y_{-}^{2}\right)\left(x_{+}^{2}+y_{+}^{2}\right)} .
\end{aligned}
$$

Note that the strip length $L$ has dropped out in Eq. (11) but not in Eq. (12). Therefore, some electrodynamic properties of long strips with applied current (e.g. their self induction) depend logarithmically on the strip length $L$, while for strips in a magnetic field usually the limit $L \rightarrow \infty$ may be taken. Strips with oblique applied field $\mathbf{H}_{a}=\left(H_{a x}, H_{a y}\right)$, or with both applied $H_{a}$ and $I_{a}$, have a lower symmetry and the integration (8) then has to be taken over the half or full cross section of the strip rather than over a quarter.

To obtain an explicit dynamic equation for the current density $j(x, y, t)$ one has to incorporate the applied vector potential $\mathbf{A}_{a}$, which for the strip may be chosen along $z$, $\mathbf{A}_{a}=A_{a}(x, y, t) \hat{\mathbf{z}}$. In general, $A_{a}(x, y, t)$ may have two parts originating from an applied perpendicular magnetic field or induction $\mathbf{B}_{a}(t)=\mu_{0} \mathbf{H}_{a}(t)=\left(B_{a x}, B_{a y}\right)$ and from an applied electric field $\mathbf{E}_{a}=E_{a}(t) \hat{\mathbf{z}}$ which drives the transport current $I_{a}$,

$$
\begin{array}{r}
A_{a}(x, y, t)=A_{a}^{B}+A_{a}^{E}, \\
\mathbf{B}_{a}=\nabla \times\left(A_{a}^{B} \hat{\mathbf{z}}\right), \quad E_{a}=-\dot{A}_{a}^{E},
\end{array}
$$

where the dot denotes the time derivative. For example, with $B_{a} \| y$ one has $A_{a}^{B}=-x B_{a}$, and with $E_{a}(t=0)=0$ one has $A_{a}^{E}=-\int_{0}^{t} E_{a}\left(t^{\prime}\right) d t^{\prime}$. Writing the total vector potential as $A=A_{j}+A_{a}$ we obtain from Eq. (8),

$$
\mu_{0} \int d^{2} r^{\prime} j\left(\mathbf{r}^{\prime}, t\right) Q_{\mathrm{bar}}\left(\mathbf{r}, \mathbf{r}^{\prime}\right)=A_{j}(\mathbf{r}, t)=A-A_{a} .
$$

For the strip the induction law $\dot{\mathbf{B}}=-\nabla \times \mathbf{E}$ yields $E=-\dot{A}$. Inserting $E$ from Eq. (5) we have for strips

$$
\dot{A}=-E_{v}(j, B)-\mu_{0} \lambda^{2} \partial j / \partial t,
$$

thus the r.h.s. of Eq. (14) may be written as

$$
A_{j}(\mathbf{r}, t)=-\int E_{v}(j, B) d t-\mu_{0} \lambda^{2} j(\mathbf{r}, t)-A_{a}(\mathbf{r}, t) .
$$

Inserting this in Eq. (14) and shifting the term $\mu_{0} \lambda^{2} j(\mathbf{r}, t)$ to the left under the integral we obtain

$$
\begin{array}{r}
\mu_{0} \int d^{2} r^{\prime} j\left(\mathbf{r}^{\prime}, t\right)\left[Q_{\mathrm{bar}}\left(\mathbf{r}, \mathbf{r}^{\prime}\right)+\lambda^{2} \delta_{2}\left(\mathbf{r}-\mathbf{r}^{\prime}\right)\right] \\
=-\int E_{v}(\mathbf{r}, t) d t-A_{a}(\mathbf{r}, t)
\end{array}
$$

where $\delta_{2}(\mathbf{r})=\delta(x) \delta(y)$ is the $2 \mathrm{D}$ delta function. Taking the time derivative $\mathrm{ff}$ Eq. (17) and introducing the inverse integral kerne 18,19

$$
K\left(\mathbf{r}, \mathbf{r}^{\prime}\right)=\left[Q_{\mathrm{bar}}\left(\mathbf{r}, \mathbf{r}^{\prime}\right)+\lambda^{2} \delta_{2}\left(\mathbf{r}-\mathbf{r}^{\prime}\right)\right]^{-1} .
$$

we arrive at the equation of motion for $j(\mathbf{r}, t)$ :

$$
\frac{\partial j(\mathbf{r}, t)}{\partial t}=-\mu_{0}^{-1} \int d^{2} r^{\prime} K\left(\mathbf{r}, \mathbf{r}^{\prime}\right)\left[E_{v}(j, B)+\dot{A}_{a}\left(\mathbf{r}^{\prime}, t\right)\right]
$$

with $\dot{A}_{a}\left(\mathbf{r}^{\prime}, t\right)=-x^{\prime} \dot{B}_{a y}(t)+y^{\prime} \dot{B}_{a x}(t)-E_{a}(t)$ and with $Q_{\text {bar }}$ from Eqs. (9), (11), or (12). In Eq. (19) only the electric field caused by the vortex motion $E_{v}$ enters explicitly. The London length $\lambda$ enters the inverse kernel $K\left(\mathbf{r}, \mathbf{r}^{\prime}\right)$ defined by

$$
\begin{array}{r}
\int d^{2} r^{\prime \prime} K\left(\mathbf{r}, \mathbf{r}^{\prime \prime}\right)\left[Q_{\mathrm{bar}}\left(\mathbf{r}^{\prime \prime}, \mathbf{r}^{\prime}\right)+\lambda^{2} \delta_{2}\left(\mathbf{r}^{\prime \prime}-\mathbf{r}^{\prime}\right)\right] \\
=\delta_{2}\left(\mathbf{r}-\mathbf{r}^{\prime}\right) .
\end{array}
$$

The kernel $K\left(\mathbf{r}, \mathbf{r}^{\prime}\right)$ may be computed by a matrix inversion as follows. First, a spatial grid $\mathbf{r}_{i}=\left(x_{i}, y_{i}\right)$ is chosen with appropriate weights $w_{i}$ such that the integrals over the strip cross section are well approximated by a sum,

$$
\int d^{2} r f(\mathbf{r}) \approx \sum_{i} f\left(\mathbf{r}_{i}\right) w_{i} .
$$

Then we express the definition (20) by such a sum,

$$
\sum_{i} K_{l i}\left(Q_{i j}^{\mathrm{bar}} w_{i}+\lambda^{2} \delta_{i j}\right)=\delta_{l j}
$$


where $Q_{i j}=Q\left(\mathbf{r}_{i}, \mathbf{r}_{j}\right)$ and $\delta_{i j}$ equals 1 if $i=j$ and 0 else. Solving Eq. (22) for the matrix $K_{i j}$ one finds

$$
K_{i j}=\left(Q_{i j}^{\mathrm{bar}} w_{i}+\lambda^{2} \delta_{i j}\right)^{-1} .
$$

The accuracy of this method is considerably increased by choosing a nonequidistant grid with narrow spacing near the specimen surface and by taking appropriate diagonal terms $Q_{i i}^{\mathrm{bar}}$ as described in the Appendix of Ref. 8a. From Eq. (23) one sees that finite $\lambda^{2}$ increases the (positive) diagonal terms of the matrix to be inverted; this makes the matrix inversion more stable.

For numerics we need the equation of motion (19) for $\mathbf{j}(\mathbf{r}, t)$ in discrete form,

$$
\frac{\partial j_{i}}{\partial t}=-\mu_{0}^{-1} \sum_{j} K_{i j}\left[E_{v}\left(j_{j}, B_{j}\right)+\dot{A}_{a j}\right],
$$

where the vectors $j_{i}(t)=j\left(\mathbf{r}_{i}, t\right), B_{i}(t)=B\left(\mathbf{r}_{i}, t\right)$, and $\dot{A}_{a i}(t)=\dot{A}_{a}\left(\mathbf{r}_{i}, t\right)=-x_{i} \dot{B}_{a y}(t)+y_{i} \dot{B}_{a x}(t)-E_{a}(t)$ are functions of the time $t$. The matrix $K_{i j}$, Eq. (23), is independent of time and has to be computed only once for a given geometry and given $\lambda$. Equation (24) is easily integrated over time $t$ starting with $j_{i}(t=0)=0$ and then switching on the applied fields $B_{a}$ and/or $E_{a}$. The resulting magnetic moment per unit length $\mathbf{m}(t)=\left(m_{x}, m_{y}\right)$ and total transport current $I_{a}(t)$ (along $z$ ) are then obtained as integrals over the strip cross section,

$$
\begin{aligned}
\mathbf{m}(t) & =\int_{-a}^{a} d x \int_{-b}^{b} d y(-\hat{\mathbf{x}} y+\hat{\mathbf{y}} x) j(x, y, t) \\
& \approx \sum_{i}\left(-\hat{\mathbf{x}} y_{i}+\hat{\mathbf{y}} x_{i}\right) j_{i}(t) w_{i} \\
I_{a}(t) & =\int_{-a}^{a} d x \int_{-b}^{b} d y j(x, y, t) \approx \sum_{i} j_{i}(t) w_{i} .
\end{aligned}
$$

Note that the contribution to $\mathbf{m}(t)$ of the U-turn of the currents at the strip ends (integrals over the $x$ and $y$ components of $\mathbf{j}$, amounting to exactly $\frac{1}{2} \mathbf{m}$ ) is already considered in Eq. (25). Note further that, though in experiments usually the applied current $I_{a}(t)$ is imposed, the theory considers a spatially constant electric field $E_{a}(t)$ along $z$ which drives the current $I_{a}(t)$ that results from the calculation.

Figures 2-6 show the current density $j(x, y)$ and the magnetic field lines of $\mathbf{B}$ (i.e. the contour lines of $A$ ) for strips with aspect ratio $b / a=0.4$ in various cases, cf. Fig. 1. In Fig. 2 the strip is in the Meissner state, i.e., no vortices have penetrated. Shown is the screening current density for finite London depth $\lambda=0.025 a$ for a strip in applied field (top) and for a strip with applied current (bottom). Similar figures for strips with quadratic cross section are depicted in Ref. 19. Note the sharp peak of $j(x, y)$ in the four corners, which has finite height. This high local current density favors the nucleation of vortex quarter loops from the corners of the strip when the applied magnetic field or current exceed a certain threshold.
Figures 3 and 4 show current density and field lines for strips in an increasing applied field $H_{a}$ for two values of the London depth $\lambda / a=0.025$ and 0.1 . Since the assumed voltage-current law is very steep, $E_{v} \propto j^{n}$ with $n=101$, a flat saturation of $j(x, y)$ occurs at the critical value $j_{c}$, like in the Bean model. The field of full penetration of this strip is $H_{p}=\left(j_{c} b / \pi\right)[(2 a / b) \arctan (b / a)+$ $\left.\ln \left(1+a^{2} / b^{2}\right)\right]=0.4945 a j_{c}$ for $b / a=0.4$ and $H_{p}=$ $2.52 \cdot\left(2 b j_{c}\right)$ for $b / a=0.001$.

Figures 5 and 6 show the same strips but with increasing applied current $I_{a}$ and with no field applied. In this case, when the critical current $I_{c}=4 a b j_{c}$ of the strip is reached, vortex rings penetrate continuously and annihilate in the center of the strip. With further increasing $I_{a}>I_{c}$, the dissipation and voltage drop increase steeply. More results for strips with both applied field and current will be published elsewhere.

Figures 7 and 8 show magnetization loops $m\left(H_{a}\right)$ of strips in perpendicular applied field $H_{a}(t)=H_{0} \sin \omega t$ at two amplitudes $H_{0}$, for five values of the London depth $\lambda$, and for two creep exponents $n$ entering in $E_{v}(j)=$ $E_{c}\left(j / j_{c}\right)^{n}$. Figure 7 is for a thick strip $(b / a=0.4)$ and Fig. 8 for a thin strip $(b / a=0.001)$. With our dimensionless units $a=j_{c}=E_{c}=\mu_{0}=1$, the used circular frequency $\omega=1$ corresponds to $\omega=E_{c} /\left(\mu_{0} j_{c} a^{2}\right)$ in physical units. Note that with increasing $\lambda$ the hysteresis loop becomes more narrow and finally collapses to a line, i.e., the magnetic response becomes reversible. This is so since with increasing $\lambda$ the screening current density decreases and can no longer depin vortices, except when $H_{a}$ is large or $n$ is small.

\section{THIN STRIPS}

This section considers the limit of thin strips, $b \ll a$, with $B_{a}$ applied perpendicular (along $y$ ) and $I_{a}$ applied parallel (along $z$ ) to the strip. In this limit only the current density integrated over the film thickness matters, called sheet current and directed along $z$ (like $j$ ),

$$
J(x, t)=\int_{-b}^{b} j(x, y, t) d y .
$$

Integrating Eq. (17) over $y$ we obtain (see also Ref. 20)

$$
\begin{array}{r}
\mu_{0} \int_{-a}^{a} d x^{\prime} J\left(x^{\prime}, t\right)\left[\frac{1}{2 \pi} \ln \frac{L}{\left|x-x^{\prime}\right|}+\Lambda \delta\left(x-x^{\prime}\right)\right] \\
=-\int E_{v}(x, t) d t-A_{a}(x, t),
\end{array}
$$

where $\Lambda=\lambda^{2} / d$ is the effective penetration depth of thin films with thickness $d=2 b<\lambda$. Here I have used the approximate constancy of the current density along $y, j(x, y) \approx J(x) / d$. Initially it was not clear to me if thin film expressions of the type (28) describe also the dynamics of superconductor strips and not only the stat$i c s$, which was successfully considered, e.g. in the static 
Bean model calculations of thin disks, 21 thin strips, $22-25$ and ellipses.26 But recently we have proven 27 that not only $j(\mathbf{r}, t)$ but also the electric field $E(\mathbf{r}, t)$ is practically constant over the film thickness; deviations from this constancy occur only near the (penetrating or exiting) flux front but are restricted to a transverse length scale of order $d$. This result was obtained in the limit $\lambda \rightarrow 0$, but it applies all the more for finite $\lambda$. Thus, the dynamics of the sheet current $J \approx j d$ is well described by Eq. (28).

Inverting Eq. (28) and taking the time derivative we obtain the equation of motion for the sheet current in thin strips,

$$
\mu_{0} \dot{J}(x, t)=-\int_{-a}^{a} d x^{\prime} K\left(x, x^{\prime}\right)\left[E_{v}\left(x^{\prime}, t\right)+\dot{A}_{a}\left(x^{\prime}, t\right)\right]
$$

with the inverse integral kernel

$$
K\left(x, x^{\prime}\right)=\left[\frac{1}{2 \pi} \ln \frac{L}{\left|x-x^{\prime}\right|}+\Lambda \delta\left(x-x^{\prime}\right)\right]^{-1}
$$

and with $E_{v}\left(x^{\prime}, t\right)=E_{v}(j, B)$ depending on $j=$ $J\left(x^{\prime}, t\right) / d$ and $B=\left|B\left(x^{\prime}, t\right)\right|$, and with $\dot{A}_{a}\left(x^{\prime}, t\right)=$ $-x^{\prime} \dot{B}_{a}(t)-E_{a}(t)$. For strips with either applied field $B_{a}$ or applied current $I_{a}$, the integration in Eq. (29) may be restricted to half the strip width, $0 \leq x \leq a$. For $B_{a} \neq 0, I_{a}=0$ one has $J(x)=-J(-x)$ and a symmetric kernel [cf. Eq. (11)]

$$
K_{B}\left(x, x^{\prime}\right)=\left[\frac{1}{2 \pi} \ln \frac{x+x^{\prime}}{\left|x-x^{\prime}\right|}+\Lambda \delta\left(x-x^{\prime}\right)\right]^{-1} .
$$

For $B_{a}=0, I_{a} \neq 0$ one has $J(x)=J(-x)$ and a symmetric kernel [cf. Eq. (12)]

$$
K_{I}\left(x, x^{\prime}\right)=\left[\frac{1}{2 \pi} \ln \frac{L^{2}}{\left|x-x^{\prime}\right|\left(x+x^{\prime}\right)}+\Lambda \delta\left(x-x^{\prime}\right)\right]^{-1} .
$$

Note that the strip length $L$ has dropped out in the kernel (31) but not in the kernel (32). The complex resistivity of thin strips thus in general depends on the logarithm of the strip length.

Figures 9 and 10 show the profiles of the sheet current $J(x)$ and perpendicular induction $B_{y}(x)$ of thin strips with increasing applied magnetic field (Fig. 9) or current (Fig. 10) for various values of the effective penetration depth $\Lambda=\lambda^{2} / d ; d=2 b$ is the strip thickness and $2 a$ the strip width, here $b / a=0.001$. Note that for larger $\Lambda$ the profiles $J(x)$ become smoother, but the bend of $J(x)$ at the point where $J$ starts to deviate from the critical sheet current $J_{c}=d j_{c}$ remains sharp. For $\Lambda / a>0.2$, the sections of $J(x)$ where $|J|<J_{c}$ are almost straight lines in Fig. 9 and almost parabolas in Fig. 10.

Figure 11 shows $J(x)$ and $B_{y}(x)$ in thin strips which are exposed to a high magnetic field $H_{a} \gg H_{p}$ before a current $I_{a}$ is applied, ranging from 0 to the critical current $I_{c}=4 a b j_{c}$. First, when $I_{a}=0$, one has $J(x) \approx J_{c} \operatorname{sign}(x)$, and finally, when $I_{a} \approx I_{c}$, one has $J(x) \approx J_{c}$ (equal signs would apply in the Bean limit $n \rightarrow \infty)$. At intermediate currents $I_{a}<I_{c}$, in the half strip $0<x<a$ one has $J \approx J_{c}=$ constant, and in the other half $-a<x<0$ the profile $J(x)$ is symmetric about the point $x=-a / 2$. For $\Lambda=0, n \gg 1$, the known Bean profiles for thipstrips with transport current apply to this half strip.22 25

\section{AXIAL SYMMETRY}

The above method for incorporating finite $\lambda$ can also be applied to axially symmetric problems, i.e. to superconductors with an axis of rotational symmetry in a magnetic field applied parallel to this axis (along y). A simple example are disks or short cylinders with radius $a$ and thickness $d=2 b$, but also other shapes like rings, toruses, cones, ellipsoids, etc. are easily described by introducing a $y$ dependent radius $a(y)$. In all these cases the current density and vector potential have only one component directed along the azimuthal unit vector $\hat{\varphi}, \mathbf{j}=j(r, y) \hat{\varphi}$ and $\mathbf{A}=A(r, y) \hat{\varphi}$, with $r=\left(x^{2}+z^{2}\right)^{1 / 2}$. Integrating the 3D Eq. (7) over the angle $\varphi=\arctan (z / x)$ we obtain the vector potential $A_{j}$ caused by the current density $j$ circulating in an axially symmetric conductor,

$$
A_{j}(r, y)=\mu_{0} \int_{-b}^{b} d y^{\prime} \int_{0}^{a\left(y^{\prime}\right)} d r^{\prime} j\left(r^{\prime}, y^{\prime}\right) Q_{\mathrm{ax}}\left(r, y ; r^{\prime}, y^{\prime}\right)
$$

with the kernell

$$
\begin{gathered}
Q_{\mathrm{ax}}\left(r, y ; r^{\prime}, y^{\prime}\right)=f\left(r, r^{\prime}, y-y^{\prime}\right), \\
f\left(r, r^{\prime}, \eta\right)=\int_{0}^{\pi} \frac{d \varphi}{2 \pi} \frac{-r^{\prime} \cos \varphi}{\left(\eta^{2}+r^{2}+r^{\prime 2}-2 r r^{\prime} \cos \varphi\right)^{1 / 2}} .
\end{gathered}
$$

When the conductor has a symmetry plane at $y=0$, then the integration over $y^{\prime}$ in Eq. (33) may be restricted to $0 \leq y \leq b$ if the kernel $Q_{\mathrm{ax}}$ is replaced by a symmetric kernel,

$$
Q_{\mathrm{cyl}}\left(r, y ; r^{\prime}, y^{\prime}\right)=f\left(r, r^{\prime}, y-y^{\prime}\right)+f\left(r, r^{\prime}, y+y^{\prime}\right) .
$$

If in addition the radius is constant (like in disks and cylinders), Eq. (33) simplifies to

$$
A_{j}(r, y)=\mu_{0} \int_{0}^{b} d y^{\prime} \int_{0}^{a} d r^{\prime} j\left(r^{\prime}, y^{\prime}\right) Q_{\mathrm{cyl}}\left(r, y ; r^{\prime}, y^{\prime}\right) .
$$

Comparing Eqs. (33) and (37) with Eqs. (8) and (10) for the strip or bar, one easily verifies that the analogue to Eq. (17) for axial symmetry is identical to Eq. (17) but with $Q_{\text {bar }}\left(\mathbf{r}, \mathbf{r}^{\prime}\right)$ replaced by $Q_{\text {ax }}\left(\mathbf{r}, \mathbf{r}^{\prime}\right)$, Eq. (34) or $Q_{\text {cyl }}\left(\mathbf{r}, \mathbf{r}^{\prime}\right)$, Eq. $(36)$, where now $\mathbf{r}=(r, y), \mathbf{r}^{\prime}=\left(r^{\prime}, y^{\prime}\right)$. In axial symmetric problems no transport current and thus no electric field is applied but only a magnetic field or induction $\mathbf{B}_{a}=\nabla \times\left(A_{a}(r, y, t) \hat{\varphi}\right)=\left(B_{a r}, B_{a y}\right)$. In general this applied field may be inhomogeneous, e.g. when levitation forces are to be computed. If $\mathbf{B}_{a}$ is homogeneous one has $A_{a}(r, y, t)=-\frac{r}{2} B_{a}(t)$. With this notation, for 
the general axially symmetric geometry the equation of motion for the circulating current density is identical to Eq. (19) but with a different inverse kernel

$$
K\left(\mathbf{r}, \mathbf{r}^{\prime}\right)=\left[Q_{\mathrm{ax}}\left(\mathbf{r}, \mathbf{r}^{\prime}\right)+\lambda^{2} \delta_{2}\left(\mathbf{r}-\mathbf{r}^{\prime}\right)\right]^{-1},
$$

with $Q_{\text {ax }}$ from Eq. (34). If the superconductor has a symmetry plane at $y=0$ and the applied field is homogeneous (or symmetric about the plane $y=0$ ) one may replace $Q_{\mathrm{ax}}$ in Eq. (38) by $Q_{\mathrm{cyl}}$ from Eq. (36),

$$
K\left(\mathbf{r}, \mathbf{r}^{\prime}\right)=\left[Q_{\text {cyl }}\left(\mathbf{r}, \mathbf{r}^{\prime}\right)+\lambda^{2} \delta_{2}\left(\mathbf{r}-\mathbf{r}^{\prime}\right)\right]^{-1},
$$

These inverse kernels $K\left(\mathbf{r}, \mathbf{r}^{\prime}\right)$ may be computed by the same method discussed below Eq. (20) if the correct integration area (or spatial grid) is used as defined in Eqs. (33) and (37). For the best choice of the diagonal terms $Q_{i i}$ see Appendix of Ref. 8a.

The axial magnetic moment of a disk or any other rotationally symmetric conductor is

$$
m(t)=\pi \int_{-b}^{b} d y \int_{0}^{a(y)} d r r^{2} j(r, y) .
$$

If the applied field is periodic, $H_{a}(t)=H_{0} \sin \omega t$, the (in general nonlinear) complex susceptibilities of the disk may be defined as $\chi_{\nu}=\chi_{\nu}^{\prime}-i \chi_{\nu}^{\prime \prime}, \nu=1,2,3 \ldots$

$$
\chi_{\nu}\left(H_{0}, \omega\right)=\frac{i}{\pi H_{0}} \int_{0}^{2 \pi} m(t) e^{-i \nu \omega t} d(\omega t) .
$$

Here $\chi_{1}$ is the fundamental susceptibility and the $\chi_{\nu}$ with $\nu>1$ correspond to higher harmonics, which are absent for linear response. The $\chi_{\nu}$ usually are normalized such that for $H_{0} \rightarrow 0$ or $\omega \rightarrow \infty$ the ideal diamagnetic susceptibility $\chi_{1}(0, \omega)=-1$ results. This is achieved by dividing all $\chi_{\nu}$, Eq. (41), by the absolute value of the initial slope $\left[\partial m\left(H_{a}\right) / \partial H_{a}\right]_{H_{a}=0}$.

As one example, Fig. 12 shows the real and imaginary parts of the nonlinear fundamental susceptibility $\chi_{1}\left(H_{0}, \omega\right)=\chi=\chi^{\prime \prime}-i \chi^{\prime}$ of a thick disk with $b / a=0.5$ plotted versus the ac amplitude $H_{0}$ for constant frequency $\omega=E_{c} /\left(\mu_{0} j_{c} a^{2}\right)$, creep exponent $n=11$, and for various London depths $\lambda / a=0.025 \ldots 1$. The same data are depicted in Fig. 13 as polar plot, $\chi^{\prime \prime}$ versus $-\chi^{\prime}$. In both presentations $\chi\left(H_{0}\right)$ sensitively depends on the parameters $n$ and $\lambda / a$ and on the geometry. More data $\chi_{\nu}\left(H_{0}, \omega\right)$ are available from the author.

\section{THIN PLATES AND FILMS}

The geometry of thin plates or planar films with arbitrary shape in a perpendicular magnetic field $H_{a} \| z$ differs from the geometries of Scts. 3 to 5 in that now the current density is no longer a scalar but has two components, $\mathbf{j}(x, y, t)=\left(j_{x}, j_{y}\right)$. However, because of the strict relation div $\mathbf{j}=0$, this planar $\mathbf{j}$ may be derived from a scalar potential (or magnetization, stream function) $g(x, y, t)$. Since we are interested in the thin film limit we consider the sheet current $\mathbf{J}(x, y, t)=\left(J_{x}, J_{y}\right)=\int \mathbf{j}(x, y, z, t) d z$, $J_{x}=\partial g / \partial y, J_{y}=-\partial g / \partial x$. The stream lines of $\mathbf{J}(x, y)$ coincide with the contour lines $g(x, y)=$ const. Thus, one may choose $g(x, y)=0$ on the edge of the film since the current flows along the edge. In this section I generalize the theory of Ref. 6 to finite London depth $\lambda$.

The magnetic field $\mathbf{H}=H_{z}(x, y) \hat{\mathbf{z}}$ in the film plane $z=0$ is related to the local magnetization $g(x, y)$ by an integral over the film area $S$,

$$
H_{z}(\mathbf{r})=H_{a}+\int_{S} d^{2} r^{\prime} Q_{\text {film }}\left(\mathbf{r}, \mathbf{r}^{\prime}\right) g\left(\mathbf{r}^{\prime}\right)
$$

with $\mathbf{r}=(x, y)$ and $\mathbf{r}^{\prime}=\left(x^{\prime}, y^{\prime}\right)$. The integral kernel

$$
Q_{\text {film }}\left(\mathbf{r}, \mathbf{r}^{\prime}\right)=\lim _{z \rightarrow 0} \frac{2 z^{2}-\rho^{2}}{4 \pi\left(z^{2}+\rho^{2}\right)^{5 / 2}}
$$

with $\rho^{2}=\left(x-x^{\prime}\right)^{2}+\left(y-y^{\prime}\right)^{2}$, gives the magnetic field generated by a magnetic dipole of unit strength positioned in the plane $z=0$ at $\left(x^{\prime}, y^{\prime}\right)$. Explicit expressions for films with rectangular shape are given by Eqs. (42) to (46) of Ref. 6 in form of Fourier series. To incorporate a finite London depth $\lambda$ we write the voltage-current law of the film in the form of Eq. (5) but now as a function of the sheet current $\mathbf{J}(x, y)=\mathbf{j}(x, y) d(x, y)$. We consider first a uniform isotropic film with constant thickness $d$. The local electric field is then

$$
\mathbf{E}(\mathbf{J}, B)=\rho_{s}(J, B) \mathbf{J}(\mathbf{r}, t)+\mu_{0} \Lambda \dot{\mathbf{J}}(\mathbf{r}, t),
$$

where $\rho_{s}=\rho / d$ is the sheet resistivity and $\Lambda=\lambda^{2} / d$ is the effective magnetic penetration depth of the film. This electric field is related to the induction $\mathbf{B}=\mu_{0} \mathbf{H}$ by the induction law $\dot{\mathbf{B}}=-\nabla \times \mathbf{E}$, which in the film plane $z=0$ means $\dot{B}_{z}=\partial E_{x} / \partial y-\partial E_{y} / \partial x$. With Eq. (44) and $J_{x}=\partial g / \partial y, J_{y}=-\partial g / \partial x$, we obtain

$$
\dot{B}_{z}(\mathbf{r}, t)=\nabla\left[\rho_{s} \nabla g(\mathbf{r}, t)\right]+\mu_{0} \Lambda \nabla^{2} \dot{g}(\mathbf{r}, t) .
$$

Taking the time derivative of Eq. (42), inserting $\dot{B}_{z}=$ $\mu_{0} \dot{H}_{z}$ from Eq. (45), and combining the two terms containing $\dot{g}$, one arrives at

$$
\begin{array}{r}
\int_{S} d^{2} r^{\prime}\left[Q_{\text {film }}\left(\mathbf{r}, \mathbf{r}^{\prime}\right)-\delta_{2}\left(\mathbf{r}-\mathbf{r}^{\prime}\right) \Lambda \nabla^{2}\right] \dot{g}\left(\mathbf{r}^{\prime}, t\right) \\
=f(\mathbf{r}, t)-\dot{H}_{a}(\mathbf{r}, t)
\end{array}
$$

with

$$
f(\mathbf{r}, t)=\mu_{0}^{-1} \nabla\left[\rho_{s}(\mathbf{r}, t) \nabla g(\mathbf{r}, t)\right] .
$$

Inverting this we obtain the equation of motion for $g(x, y, t)$ :

$$
\dot{g}(\mathbf{r}, t)=\int_{S} d^{2} r^{\prime} K\left(\mathbf{r}, \mathbf{r}^{\prime}\right)\left[f\left(\mathbf{r}^{\prime}, t\right)-\dot{H}_{a}\left(\mathbf{r}^{\prime}, t\right)\right]
$$


with $f(\mathbf{r}, t)$ from Eq. (47) and with the inverse kernel

$$
K\left(\mathbf{r}, \mathbf{r}^{\prime}\right)=\left[Q_{\text {film }}\left(\mathbf{r}, \mathbf{r}^{\prime}\right)-\delta_{2}\left(\mathbf{r}-\mathbf{r}^{\prime}\right) \Lambda \nabla^{2}\right]^{-1} .
$$

This kernel $K\left(\mathbf{r}_{i}, \mathbf{r}_{j}\right)$ may be evaluated on a grid $\mathbf{r}_{i}$ as a Fourier series with discrete $k$ vectors $\mathbf{K}$. If the Fourier coefficients of $Q_{\text {film }}\left(\mathbf{r}_{i}, \mathbf{r}_{j}\right)$ are $Q_{\mathbf{K K}^{\prime}}$, given by Eq. (46) of Ref. (6) for a rectangular platelet, then the Fourier coefficients of $K\left(\mathbf{r}_{i}, \mathbf{r}_{j}\right)$ are the inverse matrix

$$
\left[Q_{\mathbf{K K}^{\prime}}+\Lambda K^{2} \delta_{\mathbf{K K}^{\prime}}\right]^{-1}
$$

Figure 14 shows the current stream lines in a thin rectangle with side ratio $b / a=0.7$ for two effective penetration depths $\Lambda / a=0$ and $\Lambda / a=0.1$ and three applied fields $H_{a} / J_{c}=0.001,0.5$, and 1.5 (ideal screening, partial penetration, full penetration). Note that finite $\Lambda$ rounds the sharp corners of the rectangular stream lines and slightly delays vortex penetration.

If the superconductor film is nonuniform [e.g. has spatially varying thickness $d(x, y)$ ] and/or anisotropic [e.g. has two (in general nonlinear) resistivities $\rho_{x x}$ and $\rho_{y y}$ and/or two London depths $\lambda_{x}$ and $\lambda_{y}$ ], then a rather general current-voltage law $\mathbf{E}(\mathbf{J}, B, \mathbf{r})=\left(E_{x}, E_{y}\right)$ is

$$
E_{x}=\rho_{s x} J_{x}+\mu_{0} \Lambda_{x} \dot{J}_{x}, \quad E_{y}=\rho_{s y} J_{y}+\mu_{0} \Lambda_{y} \dot{J}_{y},
$$

with the two sheet resistivities

$$
\rho_{s x}(\mathbf{r})=\frac{\rho_{x x}(\mathbf{J}, B, \mathbf{r})}{d(\mathbf{r})}, \quad \rho_{s y}(\mathbf{r})=\frac{\rho_{y y}(\mathbf{J}, B, \mathbf{r})}{d(\mathbf{r})},
$$

and two effective penetration depths

$$
\Lambda_{x}(\mathbf{r})=\frac{\lambda_{x}^{2}(\mathbf{r})}{d(\mathbf{r})}, \quad \Lambda_{y}(\mathbf{r})=\frac{\lambda_{y}^{2}(\mathbf{r})}{d(\mathbf{r})} .
$$

The generalized Eqs. (45) and (46) are then

$$
\begin{gathered}
B_{z}(\mathbf{r}, t)=\frac{\partial}{\partial x}\left(\rho_{s y} \frac{\partial g}{\partial x}+\mu_{0} \Lambda_{y} \frac{\partial \dot{g}}{\partial x}\right) \\
+\frac{\partial}{\partial y}\left(\rho_{s x} \frac{\partial g}{\partial y}+\mu_{0} \Lambda_{x} \frac{\partial \dot{g}}{\partial y}\right) \\
\int_{S} d^{2} r^{\prime} \tilde{Q}\left(\mathbf{r}, \mathbf{r}^{\prime}\right) \dot{g}\left(\mathbf{r}^{\prime}, t\right)=f(\mathbf{r}, t)-\dot{H}_{a}(\mathbf{r}, t),
\end{gathered}
$$

with

$$
\begin{array}{r}
\tilde{Q}\left(\mathbf{r}, \mathbf{r}^{\prime}\right)=Q_{\text {film }}\left(\mathbf{r}, \mathbf{r}^{\prime}\right)-\delta_{2}\left(\mathbf{r}-\mathbf{r}^{\prime}\right) \\
\times\left(\frac{\partial}{\partial x} \Lambda_{y} \frac{\partial}{\partial x}+\frac{\partial}{\partial y} \Lambda_{x} \frac{\partial}{\partial y}\right) \\
f(\mathbf{r}, t)=\mu_{0}^{-1}\left[\frac{\partial}{\partial x}\left(\rho_{s y} \frac{\partial g}{\partial x}\right)+\frac{\partial}{\partial y}\left(\rho_{s x} \frac{\partial g}{\partial y}\right)\right] .
\end{array}
$$

The equation of motion for $g(x, y, t)$ now is still given by Eq. (48) but with $f(\mathbf{r}, t)$ from Eq. (57) and with the inverse kernel $K\left(\mathbf{r}, \mathbf{r}^{\prime}\right)=\tilde{Q}\left(\mathbf{r}, \mathbf{r}^{\prime}\right)^{-1}$, Eq. (56). The kernels $\tilde{Q}$ and $K$ may be evaluated by Fourier transformation.
Some figures of current stream lines and field profiles for thin rectangles with anisotropic pinning and $\Lambda=0$ are depicted in Ref. 28.

When $\Lambda=0$, the-inverse kernel $K=Q_{\text {film }}^{-1}$ can be obtained by iteration, 29 see also the Appendix of Ref. 27:

$$
K\left(\mathbf{r}, \mathbf{r}^{\prime}\right)=\frac{1}{C(\mathbf{r})}\left[\delta\left(\mathbf{r}-\mathbf{r}^{\prime}\right)+\int_{S} \frac{K\left(\mathbf{r}^{\prime \prime}, \mathbf{r}^{\prime}\right)-K\left(\mathbf{r}, \mathbf{r}^{\prime}\right)}{4 \pi\left|\mathbf{r}-\mathbf{r}^{\prime \prime}\right|^{3}} d^{2} r^{\prime \prime}\right]
$$

starting with $K\left(\mathbf{r}, \mathbf{r}^{\prime}\right)=0$. Here $C(\mathbf{r})$ is defined as an integral over the infinite area $\bar{S}$ outside the film or as a contour integral along the film edge:

$$
4 \pi C(\mathbf{r})=\int_{\bar{S}} \frac{d^{2} r^{\prime}}{R^{3}}=\int_{0}^{2 \pi} \frac{d \phi}{R(\phi)}
$$

with $R=\left|\mathbf{r}-\mathbf{r}^{\prime}\right|$. For rectangular films $(|x| \leq a,|y| \leq b)$ one has explicitly

$$
C(x, y)=\frac{1}{4 \pi} \sum_{p, q}\left[(a-p x)^{-2}+(b-q y)^{-2}\right]
$$

with $p, q= \pm 1$. Expression (60) may also be used for rectangular films with holes or slots if the integral in Eq. (58) is restricted to the area of the real film.

For numerics one needs the discretized versions of Eq. (42) and its inverse. Introducing a 2D grid $\mathbf{r}_{i}=$ $\left(x_{i}, y_{i}\right)$ with weights $w_{i}\left[\sum_{i} w_{i}=S\right.$, cf. Eq. (21)] and writing $Q_{i j}=Q\left(\mathbf{r}_{i}, \mathbf{r}_{j}\right), K_{i j}=K\left(\mathbf{r}_{i}, \mathbf{r}_{j}\right), h_{i}=H_{z}\left(\mathbf{r}_{i}\right)-$ $H_{a}$, and $g_{i}=g\left(\mathbf{r}_{i}\right)$ one has

$$
h_{i}=\sum_{j} Q_{i j} w_{j} g_{j}, \quad g_{i}=\sum_{j} K_{i j} h_{j} .
$$

The inverse kernel $K_{i j}=\left(Q_{i j} w_{j}\right)^{-1}$ cannot be obtained directly by discretizing $Q_{\text {film }}\left(\mathbf{r}, \mathbf{r}^{\prime}\right)$, Eq. (43), but it may be obtained by iterating Eq. (58). This iteration can be solved in one step by inverting a matrix,

$$
K_{i j}=\left[\delta_{i j}\left(C_{i}+\sum_{l \neq i} w_{l} q_{i l}\right)-\left(1-\delta_{i j}\right) w_{j} q_{i j}\right]^{-1},
$$

where $C_{i}=C\left(\mathbf{r}_{i}\right)$ and $q_{i j}=1 /\left(4 \pi\left|\mathbf{r}_{i}-\mathbf{r}_{j}\right|^{3}\right)$. Note that the terms in Eq. (62) must not be rearranged since $q_{i i}=\infty$.

Using this matrix $K_{i j}$ the current stream lines in ideally screening flat films of arbitrary shape or of films with trapped vortices are easily calculated. If the applied field is constant, $h_{i}=-H_{a}$, the stream lines are the contour lines of the trace of matrix $K_{i j}$ :

$$
g_{i}=-H_{a} \sum_{j} K_{i j} .
$$

If a vortex with flux $\Phi_{0}$ is trapped at a grid point $\mathbf{r}_{j}$ in the film, the magnetic field is $H_{z}(\mathbf{r})=\Phi_{0} \delta\left(\mathbf{r}-\mathbf{r}_{j}\right) \approx$ $\left(\Phi_{0} / w_{j}\right) \delta_{i j}$ at $\mathbf{r}=\mathbf{r}_{i}$, since we assume here $\Lambda=0$. Thus, $g_{i}$ is just one row of the matrix:

$$
g_{i}=\left(\Phi_{0} / w_{j}\right) \cdot K_{i j} .
$$


In general, the stream function $g_{i}$ is the linear superposition of the contributions of the applied field and trapped vortices, and possibly of currents applied by contacts.

The following relations are useful: The difference $g_{i}-g_{j}$ equals the total current crossing any line connecting the points $\mathbf{r}_{i}$ and $\mathbf{r}_{j}$; the function $\pm g(\mathbf{r})$ is the potential in which a probe vortex (or flux bundle) of appropriate sign moves; the Lorentz force on a vortex is perpendicular to the contour lines of $g(\mathbf{r})$; and the matrix $K_{i j}$ is proportional to the interaction energy between two vortices at $\mathbf{r}_{i}$ and $\mathbf{r}_{j}$. All these statements apply to any film shape and to any $\Lambda$ if the general kernel $K$, Eq. (49), is used. For $\Lambda=0$, the kernel $K$ is given by Eqs. (58) or (62).

Thus, a vortex will nucleate at the position on the film edge where the stream lines are densest, and then move to an extremum of $g(\mathbf{r})$ if it is not pinned by material inhomogeneities.

Figure 15 shows the so called washer geometry that is used for SQHDs (Superconducting Quantum Interference Devices) 30 namely, a superconductor thin square or rectangle (or circular disk) with a slot and central hole. See Refs. 3134 for theories of such washers with finite effective penetration depth $\Lambda$. After nucleation at one border of the slot, a vortex without pinning will move towards a maximum of $g(\mathbf{r})$. For the square washer in Fig. 15 (top left) there is one flat maximum of $g(\mathbf{r})$ to the left of the central hole. For the rectangular washer (bottom) there are three such maxima, to the left of the center and above and below the slot, which are separated by two saddle points above and below the central hole. A vortex may thus enter near the middle of the slot, jump to the nearby maximum of $g$, and then jump over the saddle point to the wide maximum at the left. The field-driven thermally activated motion of vortices generates low-frequency noise in the SQUID, which may be suppressed by introducing pinning centers, e.g. tiny holes 35

In the examples of Fig. $15, \Lambda=0$ (or $\Lambda \ll a$ ) was assumed. In this case the vortices interact with the screening currents of the applied field and with other vortices only via the stray field outside the film. In general, at distances $r \ll \Lambda$ the point vortices in thin films interact via a logarithmic potential similar to the interaction of line vortices in the bulk. At distances $r \gg \Lambda$ the interaction is mediated by the stray field, is of long range, and depends on the shape of the film. This long range part of the vortex interaction (or flux-bundle interaction) is given by the integral kernel $K$, Eq. (58) and (62). For infinitely extended films the $\Lambda$ dependent vortex interaction and the magnetic field of a vortex in thin films $(d \ll \lambda)$ were derived by Pearl, 12 see also Refs. 2, 36. For infinite films of arbitrary thickness the vortex interaction and magnetic field are obtained in Refs. 37.38 .

\section{SUMMARY}

In this paper finite London penetration depth $\lambda$ is introduced into known continuum methods which compute the electromagnetic response of type-II superconductors in various geometries. In addition, solution methods for some new geometries are presented, namely, thick and thin strips with transport current and with both applied current and applied magnetic field, and thin films of arbitrary shape, e.g., the washer geometry used for SQUIDs. The inverse integral kernel $K\left(\mathbf{r}, \mathbf{r}^{\prime}\right)$ or the matrix $K_{i j}$ defined in Eqs. (58) and (62) have the simple physical interpretation of the interaction energy between two point vortices in a film of given shape. The stream function $g(\mathbf{r})$ of Eqs. (63) and (64) is just the potential in which a probe vortex (or flux bundle) in the film moves and which is caused by the applied perpendicular magnetic field (and/or applied current) and by the other vortices. In the depicted case of small $\Lambda=\lambda^{2} / d$, this interaction is mediated only via the magnetic stray field outside the thin film, which crucially depends on the shape of the film. For finite films with arbitrary $\Lambda$, the vortex interaction is implicitly given by the inverse kernel $K\left(\mathbf{r}, \mathbf{r}^{\prime}\right)$, Eq. (49). Explicit $\Lambda$ dependent expressions for concrete film shapes will be given elsewhere.

\section{ACKNOWLEDGMENTS}

The author wishes to acknowledge the hospitality of the Institute of Electronic and Superconducting Materials, University of Wollongong, Australia, where part of this work was performed, and financial support from the Australian Research Council, IREX Programm.

${ }^{1}$ A. A. Abrikosov, Zh. Eksp. Teor. Fiz. 32, 1442 (1957) [Sov. Phys.-JETP 5, 1174 (1957)].

2 J. R. Clem, Phys. Rev. B 43 , 7837 (1991).

${ }^{3}$ E. H. Brandt, Rep. Prog. Phys. 58, 1465 (1995).

${ }^{4}$ E. H. Brandt, Phys. Rev. B 49, 9024 (1994).

${ }^{5}$ E. H. Brandt, Phys. Rev. B 50, 4034 (1994); E. H. Brandt, Phys. Rev. Lett. 71, 2821 (1993); E. H. Brandt, Phys. Rev. B 55, 14513 (1997).

${ }^{6}$ E. H. Brandt, Phys. Rev. B 52, 15442 (1995).

${ }^{7}$ E. H. Brandt, Phys. Rev. B 54, 4246 (1996).

${ }^{8}$ E. H. Brandt, Phys. Rev. B 58, 6506, 6523 (1998).

${ }^{9}$ L. Prigozhin, J. Computational Phys. 144, 180 (1998).

${ }^{10}$ E. H. Brandt, Phys. Rev. B 59, 3369 (1999).

${ }^{11}$ E. Zeldov, A. I. Larkin, V. B. Geshkenbein, M. Konczykowski, D. Majer, B. Khaykovich, V. M. Vinokur, and H. Shtrikman, Phys. Rev. Lett. 73, 1428 (1994).

12 J. Pearl, Appl. Phys. Lett. 5, 65 (1964).

13 J. R. Clem, Phys. Rev. Lett. 24, 1425 (1977).

${ }^{14}$ E. H. Brandt, Phys. Lett. 79A, 207 (1980); J. Low Temp. Phys. 44, 33, 59 (1981).

15 A. Perez-Gonzales and J. R. Clem, Phys. Rev. B 43, 7792 (1991), and references therein and in Ref. 3 . 
${ }^{16}$ A. Gurevich, Phys. Rev. B 46, 3638 (1992).

${ }^{17}$ E. H. Brandt, Phys. Rev. B 50, 13833 (1994).

${ }^{18}$ Here this inverse kernel is derived from the dynamic London equation (4). An alternative derivation from the static London equation $\mathbf{A}=-\mu_{0} \lambda^{2} \mathbf{j}$ is given in Ref. 19 .

${ }^{19}$ E. H. Brandt and G. P. Mikitik, Phys. Rev. Lett. 85, 4146 (2000).

${ }^{20}$ D. Yu. Vodolazov and I. L. Maximov, Physica C 349, 125 (2001).

${ }^{21}$ P. N. Mikheenko and Yu. E. Kuzovlev, Physica C 204, 229 (1994).

${ }^{22}$ W. T. Norris, J. Phys. D: Appl. Phys. 3, 498 (1970).

${ }^{23}$ E. H. Brandt, M. V. Indenbom and A. Forkl, Europhys. Lett. 22, 735 (1993).

${ }^{24}$ E. H. Brandt and M. V. Indenbom, Phys. Rev. B 48, 12893 (1993).

${ }^{25}$ E. Zeldov, J. R. Clem, M. McElfresh and M. Darwin, Phys. Rev. B 49, 9802, (1994).

${ }^{26}$ G. P. Mikitik and E. H. Brandt, Phys. Rev. B 60, 592 (1999).

${ }^{27}$ G. P. Mikitik and E. H. Brandt, Phys. Rev. B 62, 6800 (2000).

28 Th. Schuster, H. Kuhn, E. H. Brandt, and S. Klaumünzer, Phys. Rev. B 56, 3413 (1997).

${ }^{29}$ E. H. Brandt, Phys. Rev. B 46, 8628 (1992).

${ }^{30}$ D. Koelle, R. Kleiner, F. Ludwig, E. Dantsker, and John Clarke, Rev. Mod. Phys. 71, 631 (1999).

${ }^{31}$ H. W. Chang, IEEE Trans. Magn. 17, 764 (1981).

${ }^{32}$ M. B. Ketchen, W. J. Gallagher, A. W. Kleinsasser, S. Murphy, and J. R. Clem, in: SQUID '85, Superconducting Quantum Interference Devices and their Applications, H. D. Hahlbohm and H. Lübbig, eds. (de Gruyter, Berlin 1985), pp. 865-871.

${ }^{33}$ G. Hildebrandt and F. H. Uhlmann, IEEE Trans. Magn. 32, 690 (1996).

${ }^{34}$ M. M. Kapaev, Supercond. Sci. Technol. 10, 389 (1997).

${ }^{35}$ P. Selders and R. Wördenweber, Appl. Phys. Lett. 76, 3277 (2000).

${ }^{36}$ E. Olive and E. H. Brandt, Phys. Rev. B 59, 7116 (1999).

37 J.-C. Wei and T.-J. Yang, Jpn. J. Appl. Phys., Part 1 35, 5696 (1996).

${ }^{38}$ G. Carneiro and E. H. Brandt, Phys. Rev. B 61, 6370 (2000).
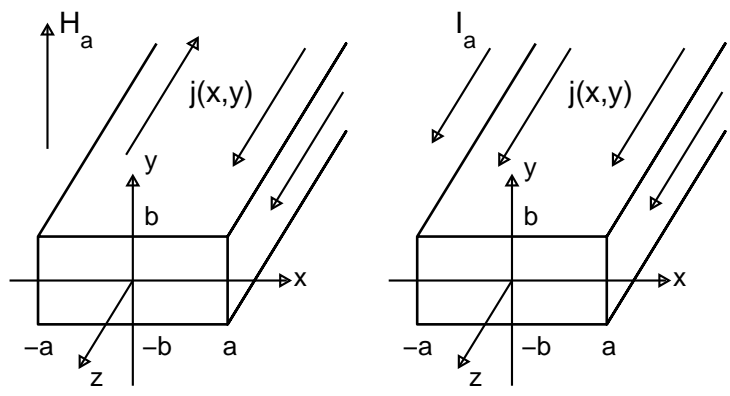

FIG. 1. Geometry of long superconductor strips with rectangular cross section $2 a \times 2 b$ in a perpendicular applied magnetic field $H_{a}$ (left) or with applied electric current $I_{a}=I=\int j(x, y) d x d y$ (right).
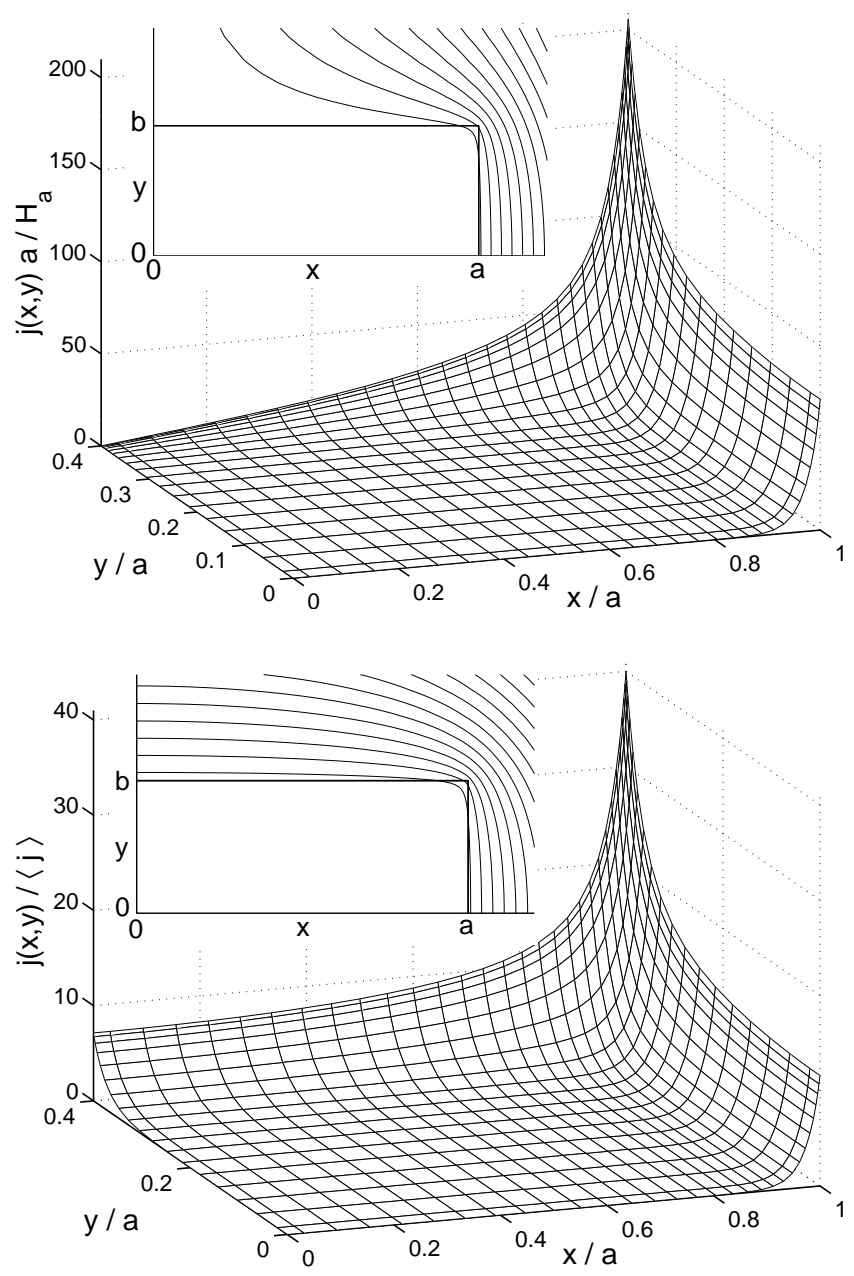

FIG. 2. A strip with aspect ratio $b / a=0.4$, cf. Fig. 1, in the Meissner state with London penetration depth $\lambda=0.025 a$. Shown is the current density in a quarter of the cross section and the magnetic field lines (inset). Top: In perpendicular applied magnetic field $H_{a}$. Bottom: With applied current. 

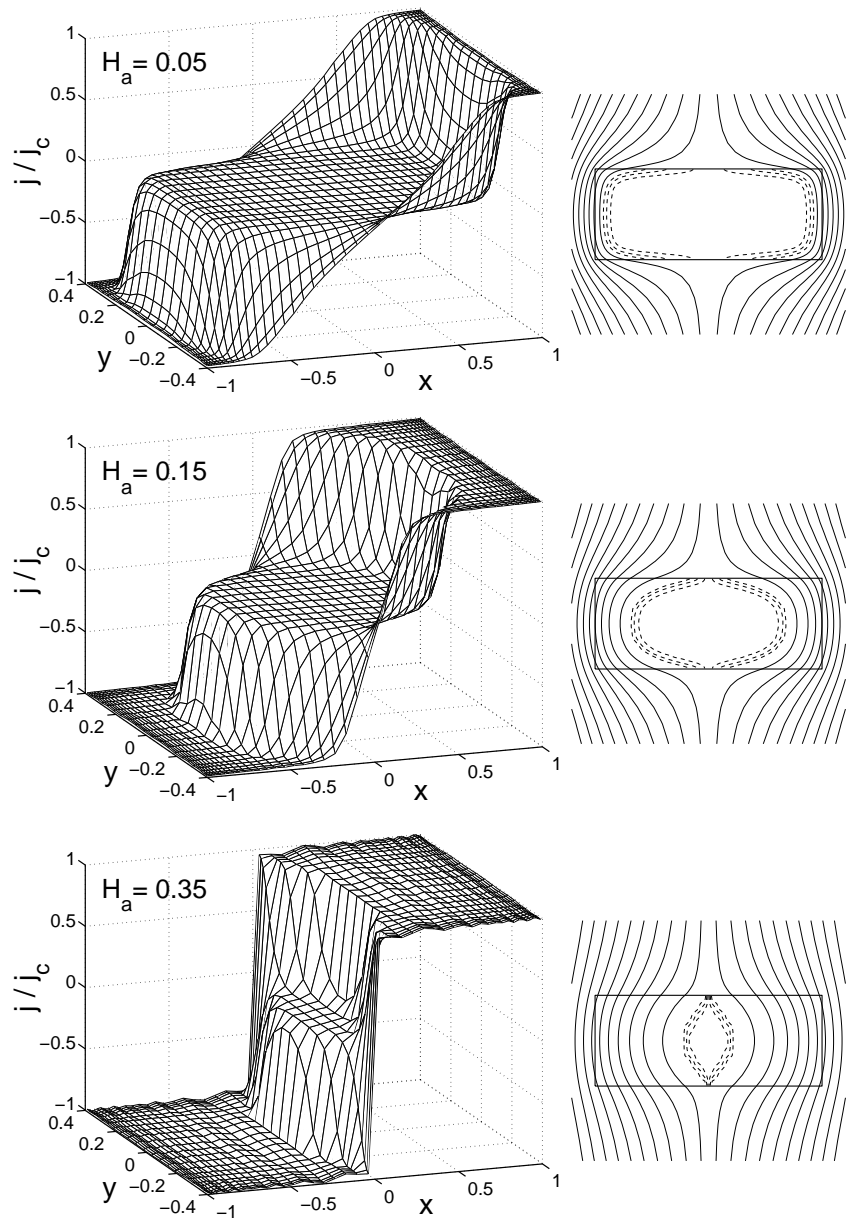

FIG. 3. Current density $j(x, y)$ (left) and magnetic field lines (right) of a superconductor strip with aspect ratio $b / a=0.4$ in increasing magnetic field $H_{a}=0.05,0.15$, and 0.35 in units $a j_{c}\left(j_{c}=\right.$ critical current density). The dashed lines are contours of the current density at $j / j_{c}= \pm 0.75$, \pm 0.45 , and \pm 0.15 . The superconductor is characterized by a pinning-caused voltage-current law $E \propto\left(j / j_{c}\right)^{101}$ and by a small London penetration depth $\lambda / a=0.025$.
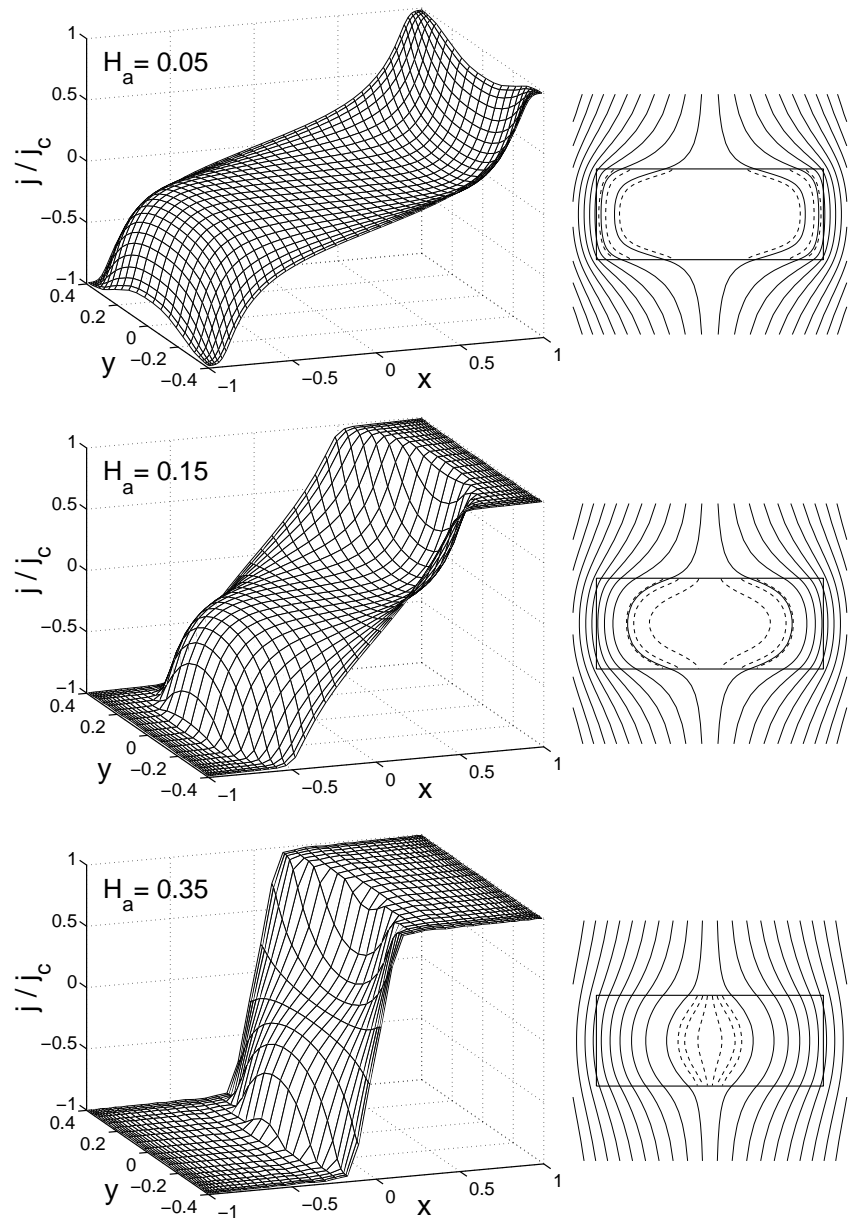

FIG. 4. As Fig. 3 but for larger London depth $\lambda / a=0.1$. 

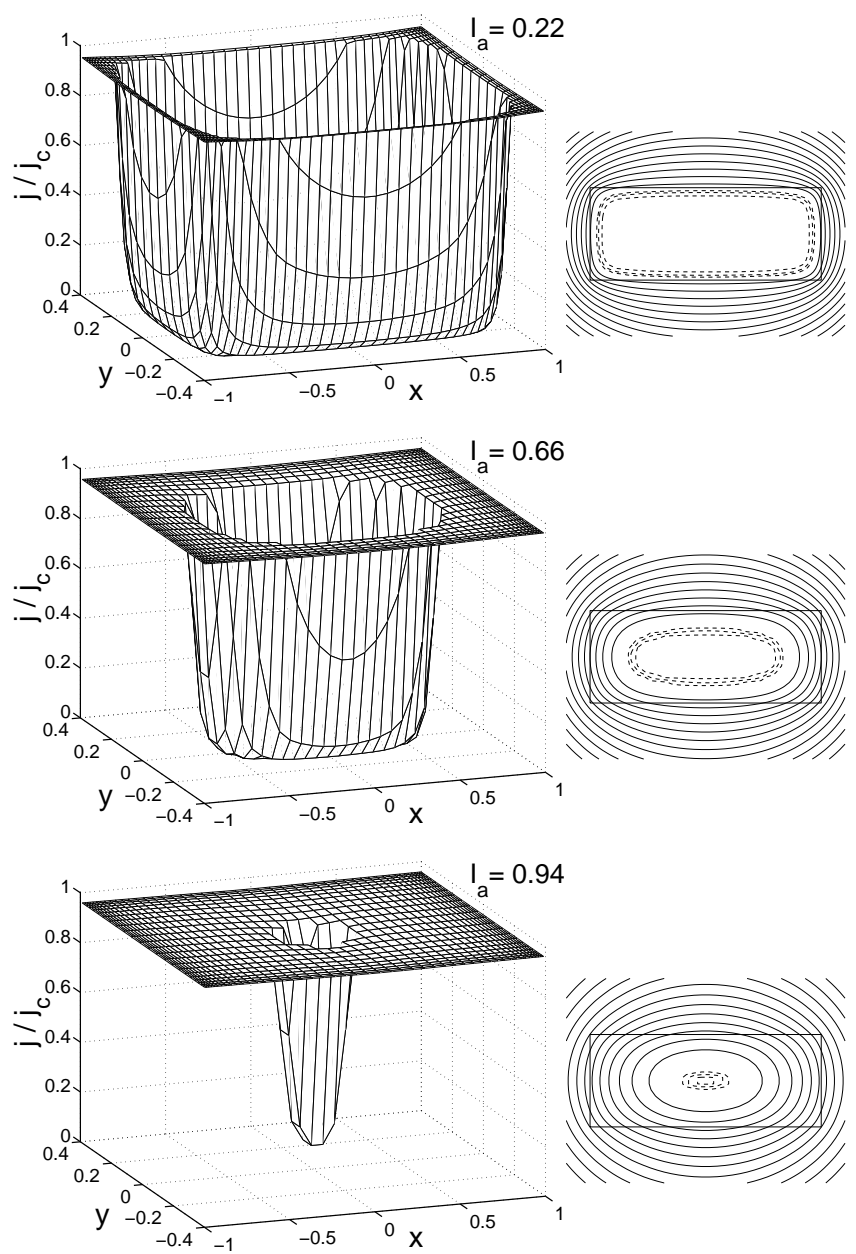

FIG. 5. Current density $j(x, y)$ (left) and magnetic field lines (right) of a superconductor strip with aspect ratio $b / a=0.4$ with applied current $I_{a}=0.22,0.66$, and 0.94 in units of the critical current $I_{c}=4 a b j_{c}$. The dashed lines are contours of the current density at $j / j_{c}=0.75,0.45$, and 0.15. As in Figs. 3, 4 a voltage-current law $E \propto\left(j / j_{c}\right)^{101}$ was used and a small London penetration depth $\lambda / a=0.025$.
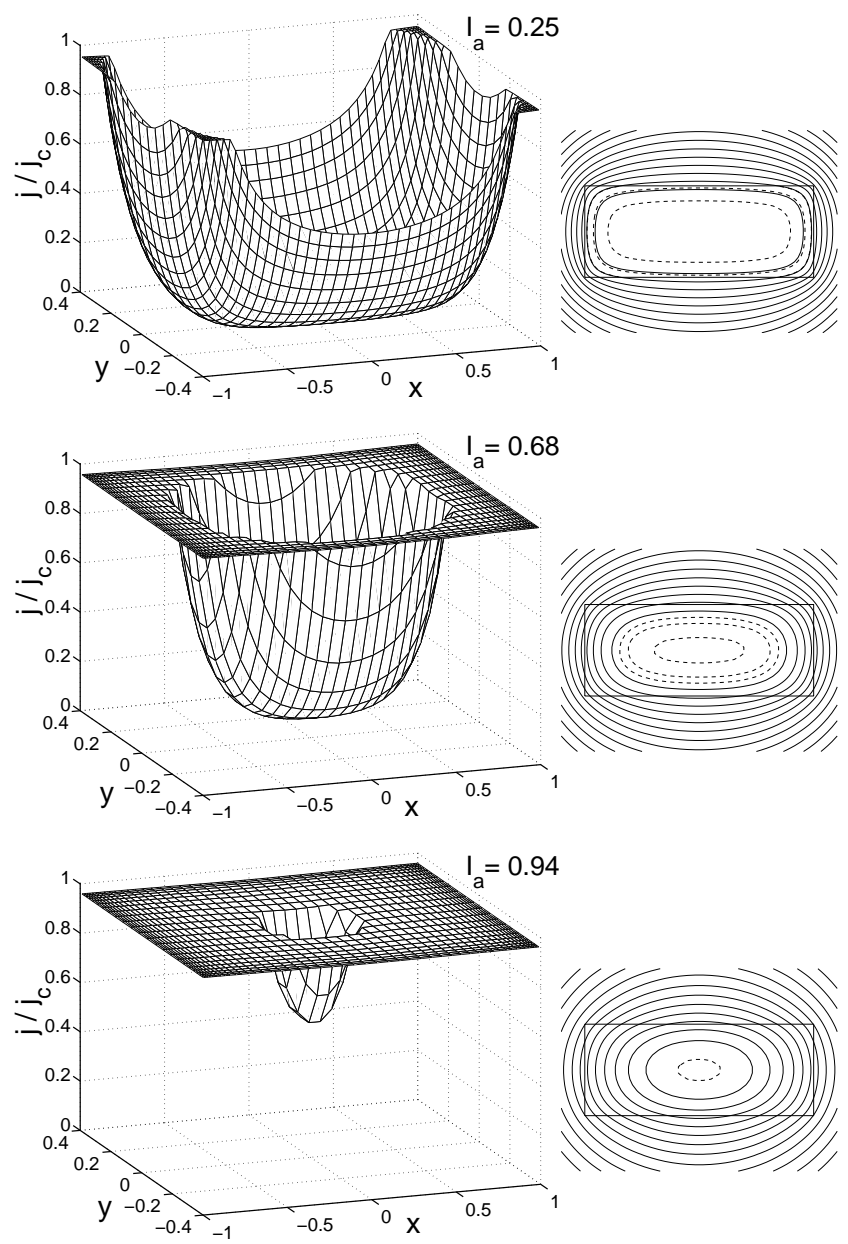

FIG. 6. As Fig. 5 but for larger London depth $\lambda / a=0.1$. 

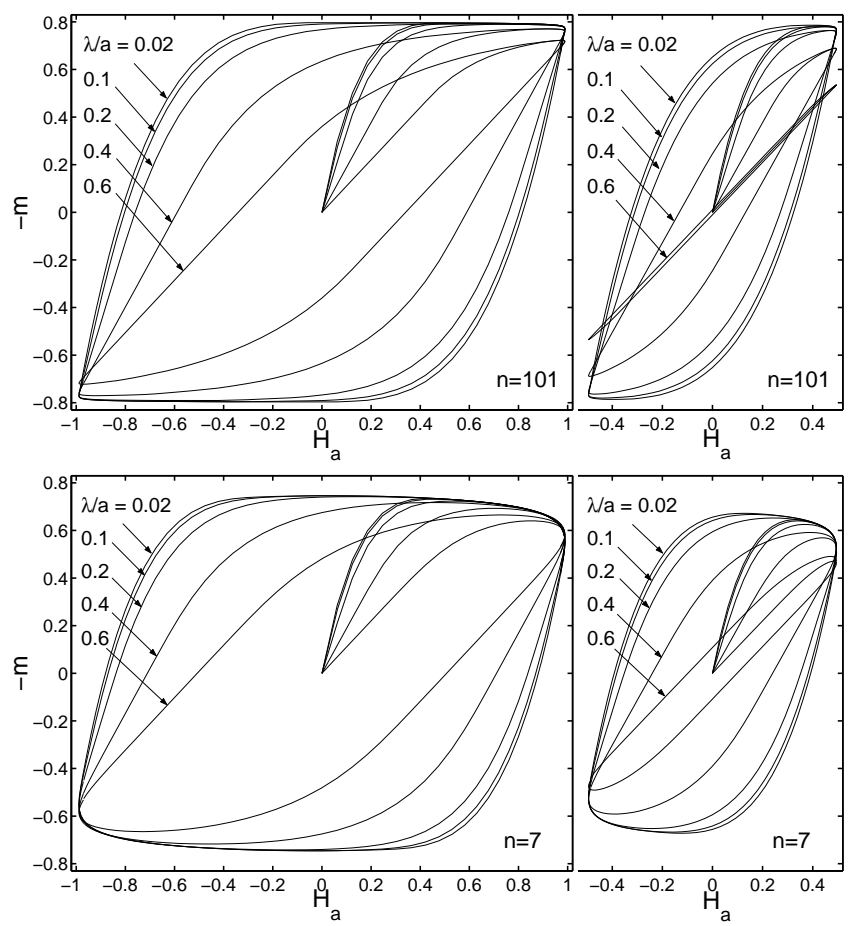

FIG. 7. Magnetization curves $m\left(H_{a}\right)$ of a superconductor thick strip with aspect ratio $b / a=0.4$ in a perpendicular field $H_{a}(t)=H_{0} \sin \omega t$ at two amplitudes $H_{0} / H_{p}=2$ (left) and $H_{0} / H_{p}=1$ (right) with $H_{p}=0.4945 a j_{c}$ the field of full penetration. Fields in units $a j_{c}$ and magnetic moment $m$ in units $a^{3} j_{c}$ (per unit length). Shown are virgin curves and hysteresis loops for two creep exponents $n=101$ (top) and $n=7$ (bottom) and for London depths $\lambda / a=0.02,0.1,0.2,0.4$, and 0.6 .
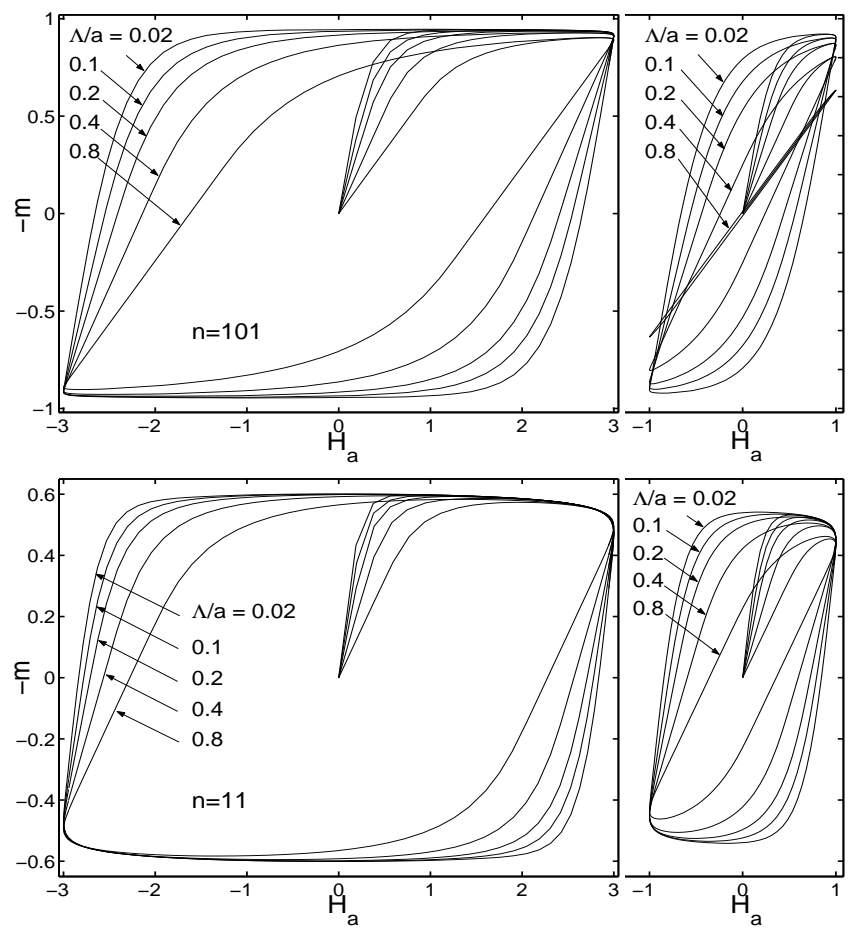

FIG. 8. Magnetization curves $m\left(H_{a}\right)$ of a superconductor thin strip with aspect ratio $b / a=0.001$ in a perpendicular field $H_{a}(t)=H_{0} \sin \omega t$ at two amplitudes $H_{0}=3$ (left) and $H_{0}=1$ (right) in units of the critical sheet current $J_{c}=d j_{c}$ $(d=2 b)$. Magnetic moment $m$ in units $a^{2} J_{c}$ (per unit length), $H_{p}=2.52 J_{c}$. Virgin curves and hysteresis loops for two creep exponents $n=101$ (top) and $n=11$ (bottom) and five effective penetration depths $\Lambda=\lambda^{2} / d: \Lambda / a=0.02,0.1,0.2,0.4$, and 0.8 

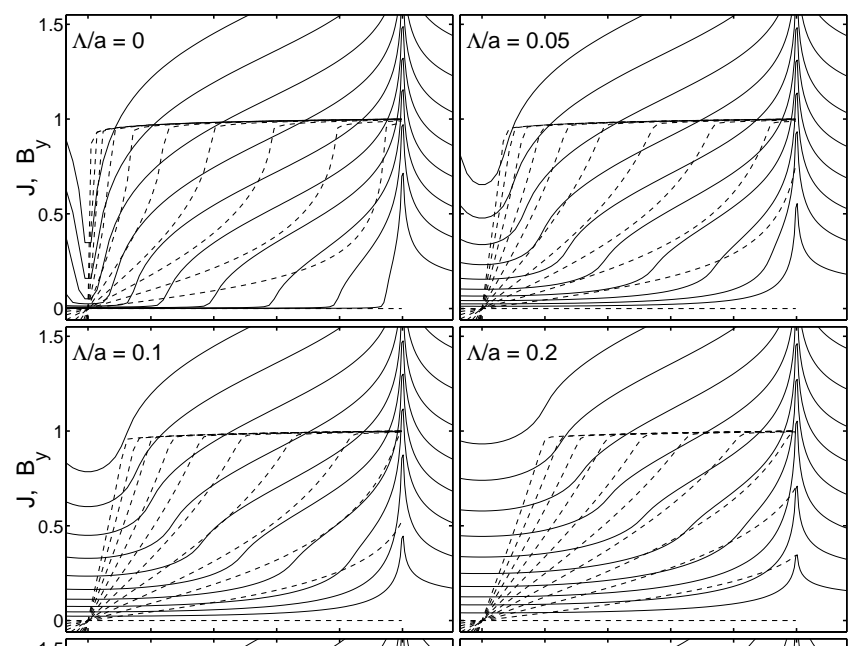

$\Lambda / a=0.2$
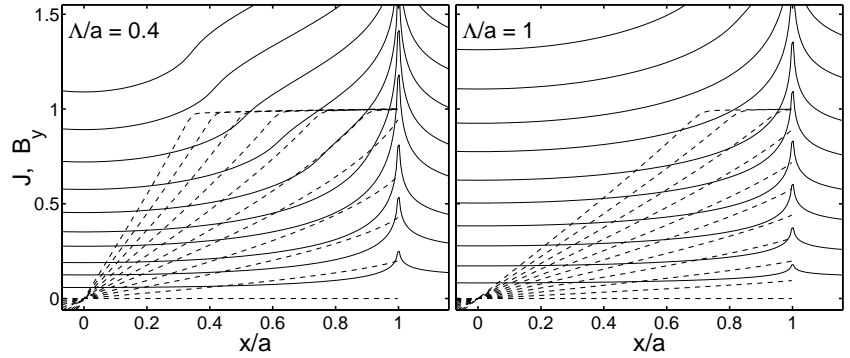

FIG. 9. Profiles of sheet current $J(x)$ (dashed lines) and perpendicular induction $B_{y}(x)$ (solid lines) of a thin strip in increasing perpendicular magnetic field $H_{a}=0.10,0.22,0.35$, $0.49,0.65,0.82,1.0,1.23,1.46$, and 1.72 (curves from bottom to top). $J$ and $H_{a}$ are in units $J_{c}=d j_{c}(d=2 b \ll a), B$ in units $\mu_{0} J_{c}$. For six effective penetration depths $\Lambda=\lambda^{2} / d$ : $\Lambda / a=0,0.05,0.1,0.2,0.4$, and 1 . Creep exponent $n=51$.

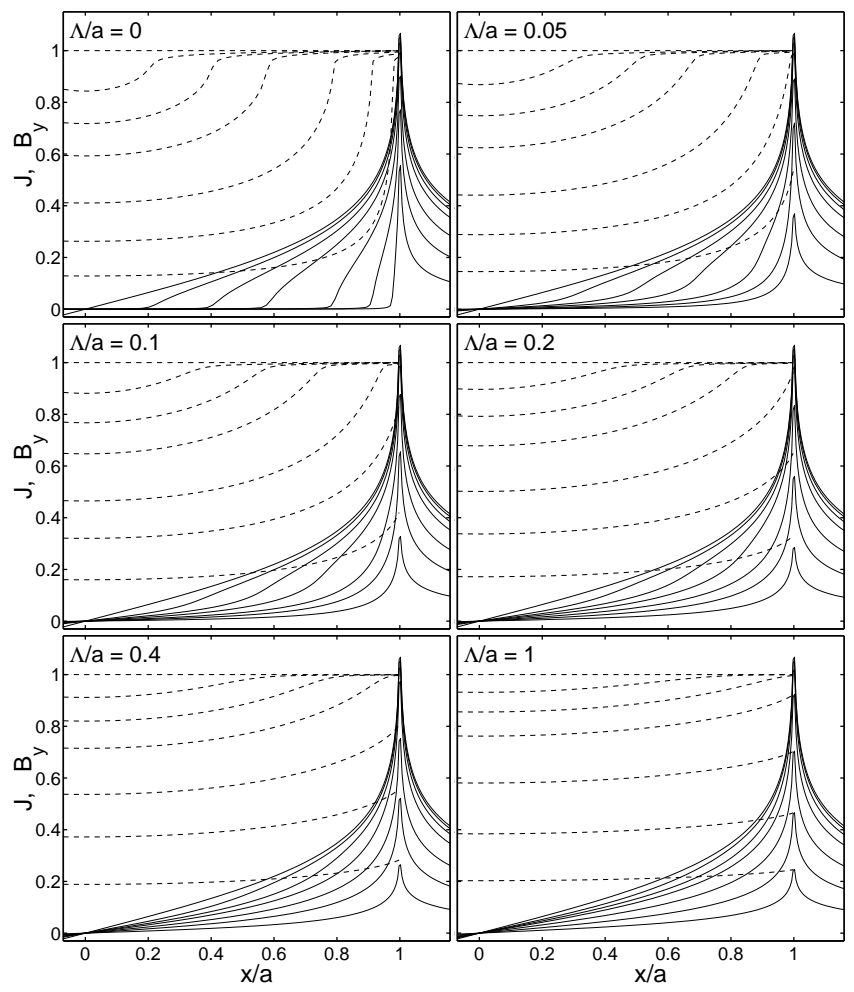

FIG. 10. As Fig. 9 but for strips with increasing applied current $I_{a} / I_{c}=0,0.2,0.4,0.6,0.8,0.9,0.965$, and 1 . $I_{c}=4 a b j_{c}$ is the critical current of the strip.
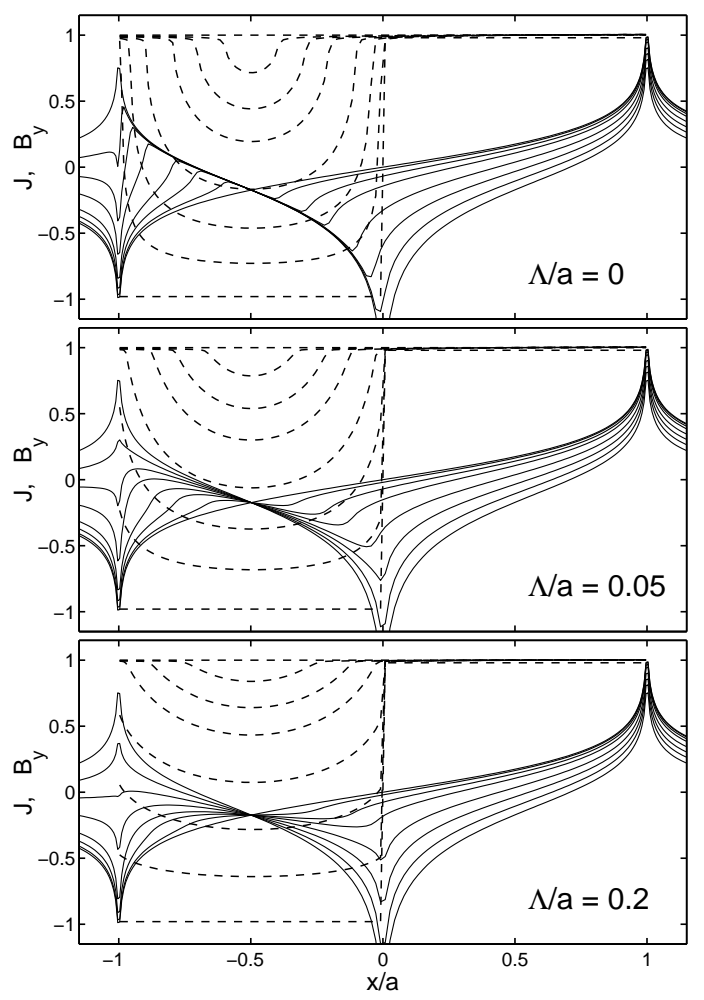

FIG. 11. As Fig. 9 but for a thin strip in large magnetic field $H_{a} \gg H_{p}$ to which an increasing current is applied, $I_{a} / I_{c}=0,0.2,0.4,0.6,0.8,0.9,0.97$, and 1 (from bottom to top) with $I_{c}=4 a b j_{c} . \Lambda / a=0,0.05$, and 0.2. $n=51$. 


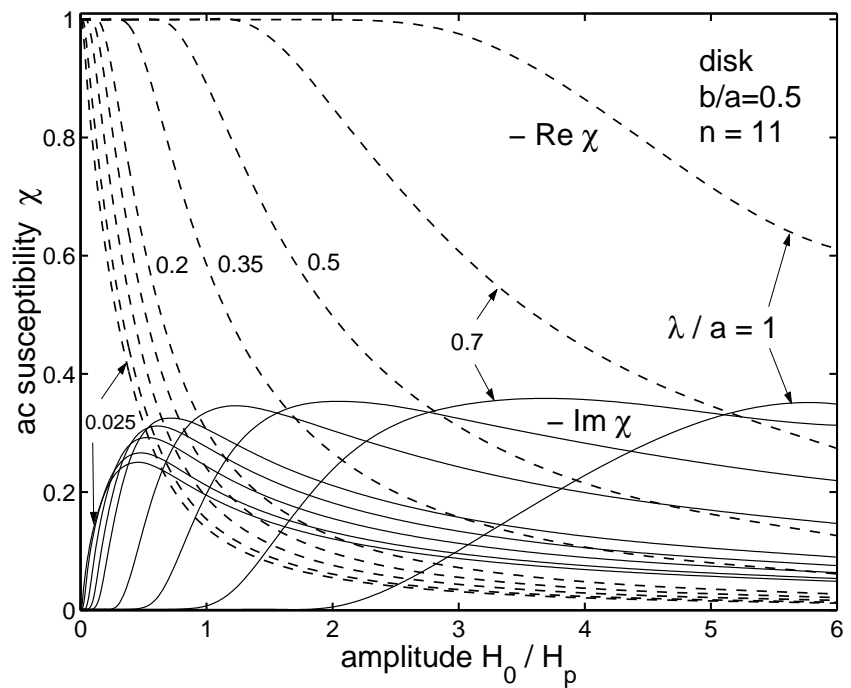

FIG. 12. The nonlinear complex ac susceptibility $\chi\left(H_{0}, \omega\right)=\chi^{\prime}-i \chi^{\prime \prime}$ of a type-II superconductor thick disk with radius $a$ and half thickness $b=0.5 a$ plotted versus the amplitude $H_{0}$ of the ac magnetic field in units of the Bean field of full penetration $H_{p}=0.722 a j_{c}$. The curves are for different London depths $\lambda / a=0.025,0.05,0.1,0.15,0.2,0.35$, $0.5,0.7$, and 1. $E_{v} \propto j^{n}, n=11, \omega=E_{c} /\left(\mu_{0} j_{c} a^{2}\right)$.

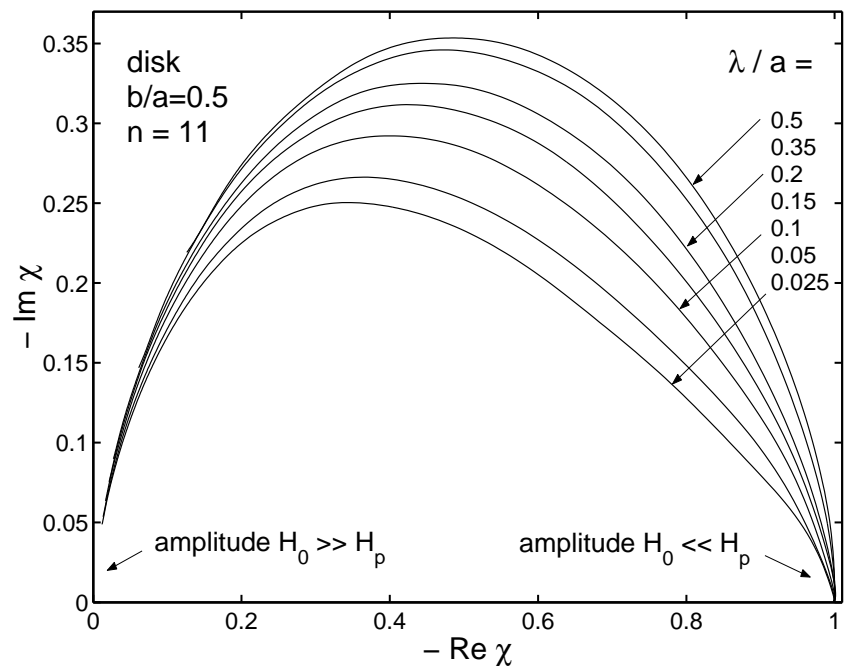

FIG. 13. The nonlinear susceptibility of Fig. 12 plotted as $\chi^{\prime \prime}$ versus $-\chi^{\prime}$.

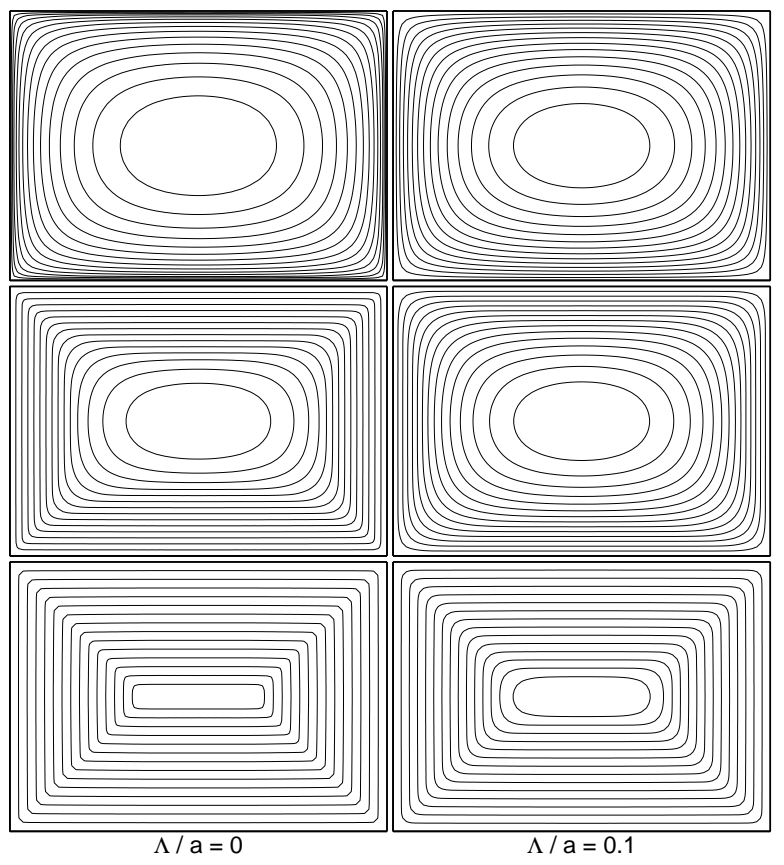

FIG. 14. The current stream lines in a thin superconductor rectangle $(b / a=0.7)$ with $\Lambda / a=0$ (left) and $\Lambda / a=0.1$ (right) at applied perpendicular fields (from top to bottom) $H_{a} / J_{c}=0.001,0.5$, and 1.5 .

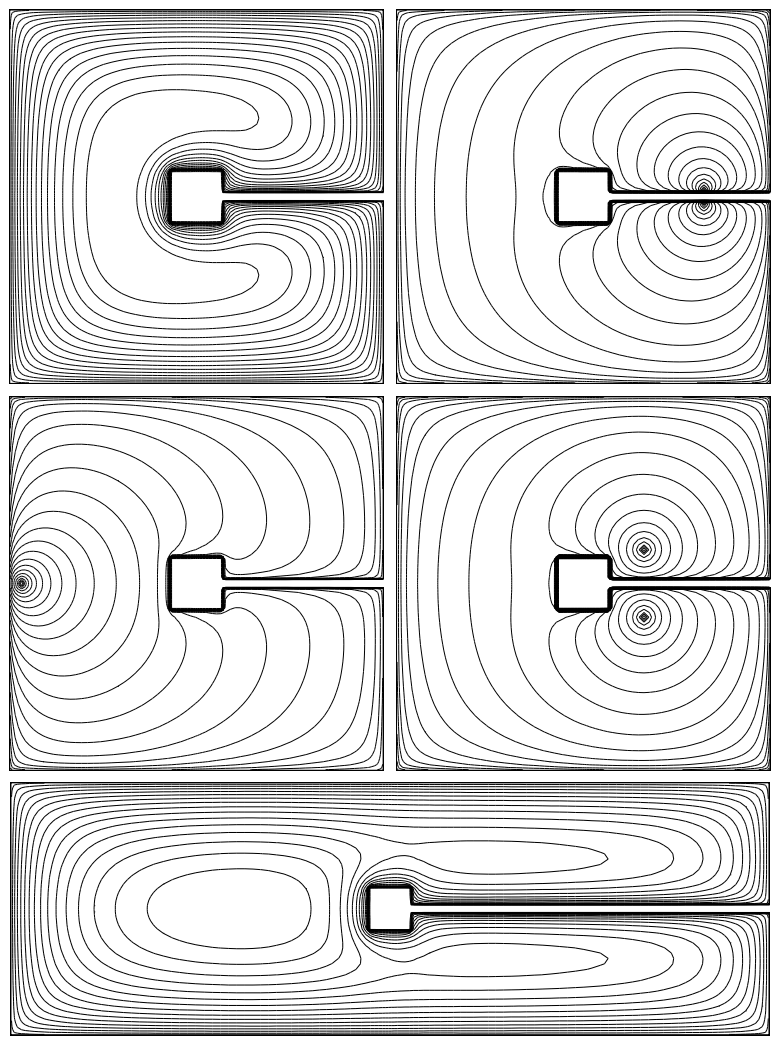

FIG. 15. Thin superconductor square $(a=b)$ and rectangle $(a / b=3)$ with slit and hole (washer) in the Meissner state with $\Lambda=0$. Shown are the current stream lines for constant applied field (top left, bottom) and for zero applied field but with one or two vortices trapped at various positions. 